\title{
The Maximum k-Differential Coloring Problem औै
}

\author{
Michael A. Bekos ${ }^{\mathrm{a}}$, Michael Kaufmann ${ }^{\mathrm{a}}$, Stephen G. Kobourov ${ }^{\mathrm{b}}$, \\ Konstantinos Stavropoulos ${ }^{\mathrm{c}}$, Sankar Veeramoni ${ }^{\mathrm{b}}$ \\ ${ }^{a}$ Wilhelm-Schickard-Institut für Informatik - Universität Tübingen, Germany \\ $\{$ bekos,mk\}@informatik.uni-tuebingen.de \\ ${ }^{b}$ Department of Computer Science - University of Arizona, Tucson AZ, USA \\ $\{$ kobourov,sankar\}@cs.arizona.edu \\ ${ }^{c}$ RWTH Aachen University, Aachen, Germany \\ stavropoulos@cs.rwth-aachen.de
}

\begin{abstract}
Given an $n$-vertex graph $G$ and two positive integers $d, k \in \mathbb{N}$, the $(d, k n)$-differential coloring problem asks for a coloring of the vertices of $G$ (if one exists) with distinct numbers from 1 to $k n$ (treated as colors), such that the minimum difference between the two colors of any adjacent vertices is at least $d$. While it was known that the problem of determining whether a general graph is $(2, n)$-differential colorable is NPcomplete, our main contribution is a complete characterization of bipartite, planar and outerplanar graphs that admit $(2, n)$-differential colorings. For practical reasons, we also consider color ranges larger than $n$, i.e., $k>1$. We show that it is NP-complete to determine whether a graph admits a $(3,2 n)$-differential coloring. The same negative result holds for the $(\lfloor 2 n / 3\rfloor, 2 n)$-differential coloring problem, even in the case where the input graph is planar.
\end{abstract}

\section{Introduction}

Several methods for visualizing relational datasets use a map metaphor where objects, relations between objects and clusters are represented as cities, roads and countries, respectively. Clusters are usually represented by colored regions, whose boundaries are explicitly defined. The 4-coloring theorem states that four colors always suffice to color any map such that neighboring countries have distinct colors. However, if not all countries of the map are contiguous and the countries are not colored with unique colors, it would be impossible to distinguish whether two regions with the same color belong to the same country or to different countries. In order to avoid such ambiguity, this necessitates the use of a unique color for each country; see Fig. 1

\footnotetext{
This article is based on the short version: [Michael A. Bekos, Michael Kaufmann, Stephen G. Kobourov and Sankar Veeramoni: The Maximum k-Differential Coloring Problem. In G. F. Italiano ed., Proc. of 41st International Conference on Current Trends in Theory and Practice of Computer Science (SOFSEM2015), p.p. 115-127, LNCS 8939, 2015.]
} 


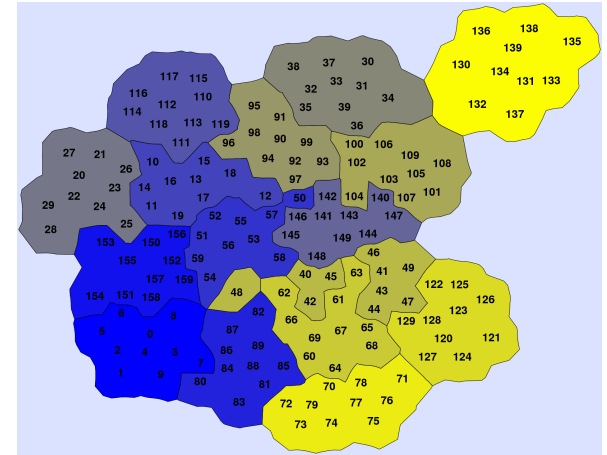

(a) Colored with random assignment of colors

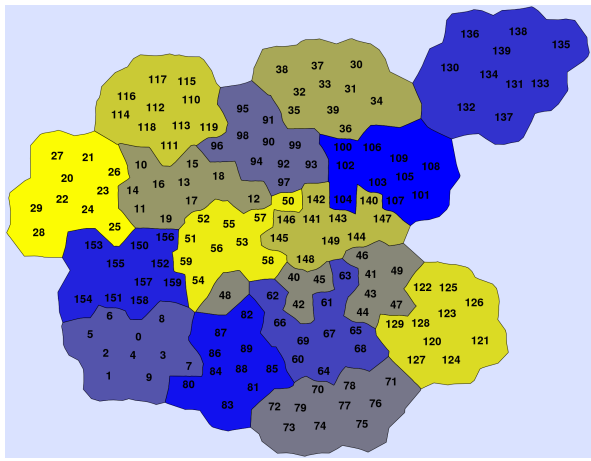

(b) Colored with max. differential coloring

Figure 1: Illustration of a map colored using the same set of colors obtained by the linear interpolation of blue and yellow. There is one country in the middle containing the vertices 40-49 which is fragmented into three small regions.

However, it is not enough to just assign different colors to each country. Although human perception of color is good and thousands of different colors can be easily distinguished, reading a map can be difficult due to color constancy and color context effects [1]. Dillencourt et al. [2] define a good coloring as one in which the colors assigned to the countries are visually distinct while also ensuring that the colors assigned to adjacent countries are as dissimilar as possible. However, not all colors make suitable choices for coloring countries [3] and a "good" color palette is often a gradation of certain map-like colors [4]. In more restricted scenarios, e.g., when a map is printed in gray scale, or when the countries in a given continent must use different shades of a predetermined color, the color space becomes 1-dimensional.

This 1-dimensional fragmented map coloring problem is nicely captured by the maximum differential coloring problem [5, 6, 7, 8], which we slightly generalize in this paper. Given a map, define the country graph $G=(V, E)$ in which vertices represent countries and two vertices are connected by an edge if their corresponding countries share a non-trivial geographic boundary. Given two positive integers $d, k \in \mathbb{N}$, we say that $G$ is $(d, k n)$-differential colorable if and only if there is a coloring of the $n$ vertices of $G$ with distinct numbers from 1 to $k n$ (treated as colors), so that the minimum color distance between adjacent vertices of $G$ is at least $d$. The maximum $k$-differential coloring problem asks for the largest value of $d$, called the $k$-differential chromatic number of $G$, so that $G$ is $(d, k n)$-differential colorable. Note that the traditional maximum differential coloring problem corresponds to $k=1$.

A natural reason to study the maximum k-differential coloring problem for $k>1$ is that using more colors can help produce maps with larger differential chromatic number. Note, for example, that a star graph on $n$ vertices has 1-differential chromatic number (or simply differential chromatic number) one, whereas its 2-differential chromatic number is $n+1$. That is, by doubling the number of colors used, we can improve the quality of the resulting coloring by a factor of $n$. This is our main motivation for studying the maximum k-differential coloring problem for $k>1$. 


\subsection{Related Work}

The maximum differential coloring problem is a well-studied problem, which dates back in 1984, when Leung et al. [6] introduced it under the name "separation number" and showed its NP-completeness. It is worth mentioning though that the maximum differential coloring problem is also known as "dual bandwidth" [8] and "antibandwidth" [5], since it is the complement of the bandwidth minimization problem [9]. Due to the hardness of the problem, heuristics are often used for coloring general graphs, e.g., LP-formulations [10], memetic algorithms [11] and spectral based methods [12]. The differential chromatic number is known only for special graph classes, such as Hamming graphs [13], meshes [14], hypercubes [14, 15], complete binary trees [16], complete $m$-ary trees for odd values of $m$ [5], other special types of trees [16], and complements of interval graphs, threshold graphs and arborescent comparability graphs [17]. Upper bounds on the differential chromatic number are given by Leung et al. [6] for connected graphs and by Miller and Pritikin [7] for bipartite graphs. Note that closely related is also the radio frequency assignment problem, where $n$ transmitters have to be assigned $n$ frequencies, so that interfering transmitters have frequencies as far apart as possible, see e.g., [18, 19]. For a more detailed bibliographic overview related to the maximum differential coloring problem refer to [20].

\subsection{Our Contribution}

In Section 2, we present preliminary properties and bounds on the $k$-differential chromatic number, for $k \geq 1$. One of them guarantees that any graph is $(1, n)$ differential colorable; an arbitrary assignment of distinct colors to the vertices of the input graph guarantees a minimum color distance of one (see Lemma11). So, the next reasonable question to ask is whether a given graph is $(2, n)$-differential colorable. Unfortunately, this is already an NP-complete problem (for general graphs), since a graph is $(2, n)$-differential colorable if and only if its complement has a Hamiltonian path [6]. This motivates the study of the $(2, n)$-differential coloring problem for special classes of graphs. In Section 3, we present a complete characterization of bipartite, outer-planar and planar graphs that admit $(2, n)$-differential colorings.

In Section 4, we double the number of available colors. As any graph is $(2,2 n)$ differential colorable (due to Lemma11, Section 2), we study the $(3,2 n)$-differential coloring problem and we prove that it is NP-complete for general graphs (Theorem 4 , Section 47. More general, we show that for any given $k \geq 2$ testing whether a given graph is $(k+1, k n)$-differential colorable is NP-complete (Theorem 5, Section 4). On the other hand, all planar graphs are $(\lfloor n / 3\rfloor+1,2 n)$-differential colorable (see Lemma 3 , Section 2$)$ and testing whether a given planar graph is $(\lfloor 2 n / 3\rfloor, 2 n)$-differential colorable is shown to be NP-complete (Theorem 6, Section 4). In Section 5, we provide a simple ILP-formulation for the maximum k-differential coloring problem and experimentally compare the optimal results obtained by the ILP formulation for $k=1$ and $k=2$ with the GMap coloring method, which relies on a heuristic based on spectral methods [21]. We conclude in Section 6 with open problems and future work. 


\section{Preliminaries}

We assume familiarity with basic concepts of graph theory and refer to the textbooks [22] for background. The maximum k-differential coloring problem can be easily reduced to the ordinary differential coloring problem as follows: Consider the maximum k-differential coloring of an $n$-vertex graph $G$; create a (disconnected) graph $G^{\prime}$ that contains all vertices and edges of $G$ plus $(k-1) n$ isolated vertices. It is easy to see that the k-differential chromatic number of $G$ is equal to the 1-differential chromatic number of $G^{\prime}$. A drawback of this approach, however, is that few results are known for the ordinary differential coloring problem, when the input is a disconnected graph. In the following, we present some immediate upper and lower bounds on the $k$-differential chromatic number for connected graphs.

Lemma 1. For $k \geq 1$, the $k$-differential chromatic number of a connected graph is at least $k$.

Proof. Let $G$ be a connected graph on $n$ vertices. It suffices to prove that $G$ is $(k, k n)$ differential colorable. Indeed, an arbitrary assignment of distinct colors from the set $\{k, 2 k, \ldots, k n\}$ to the vertices of $G$ guarantees a minimum color distance of $k$.

Lemma 2. For $k \geq 1$, the $k$-differential chromatic number of a connected graph $G=(V, E)$ on $n$ vertices is at most $\left\lfloor\frac{n}{2}\right\rfloor+(k-1) n$.

Proof. The proof is a straightforward generalization of the proof of Yixun and Jinjiang [8] for the ordinary maximum differential coloring problem. One of the vertices of $G$ has to be assigned with a color in the interval $\left[\left\lceil\frac{n}{2}\right\rceil,\left\lceil\frac{n}{2}\right\rceil+(k-1) n\right]$, as the size of this interval is $(k-1) n+1$ and there can be only $(k-1) n$ unassigned colors. Since $G$ is connected, that vertex must have at least one neighbor which (regardless of its color) would make the difference along that edge at most $k n-\left\lceil\frac{n}{2}\right\rceil=\left\lfloor\frac{n}{2}\right\rfloor+(k-1) n$.

Lemma 3. For $k \geq 1$, the $k$-differential chromatic number of a connected $m$-colorable graph $G=(V, E)$ on $n$ vertices is at least $\left\lfloor\frac{(k-1) n}{m-1}\right\rfloor+1$.

Proof. Let $C_{i} \subseteq V$ be the set of vertices of $G$ with color $i$ and $c_{i}$ be the number of vertices with color $i, i=1, \ldots, m$. We can show that $G$ is $\left(\left\lfloor\frac{(k-1) n}{m-1}\right\rfloor+1, k n\right)$ differential colorable by coloring the vertices of $C_{i}$ with colors from the following set: $\left[\left(\sum_{j=1}^{i-1} c_{j}\right)+1+(i-1)\left\lfloor\frac{(k-1) n}{m-1}\right\rfloor,\left(\sum_{j=1}^{i} c_{j}\right)+(i-1)\left\lfloor\frac{(k-1) n}{m-1}\right\rfloor\right]$

\section{The (2,n)-Differential Coloring Problem}

In this section, we provide a complete characterization of (i) bipartite graphs, (ii) outerplanar graphs and (iii) planar graphs that admit $(2, n)$-differential coloring. Central to our approach is a result of Leung et al. [6] who showed that a graph $G$ has $(2, n)$-differential coloring if and only if the complement $G^{c}$ of $G$ is Hamiltonian. As a consequence, if the complement of $G$ is disconnected, then $G$ has no $(2, n)$-differential coloring.

In order to simplify our notation scheme, we introduce the notion of ordered differential coloring (or simply ordered coloring) of a graph, which is defined as follows. 
Given a graph $G=(V, E)$ and a sequence $S_{1} \rightarrow S_{2} \rightarrow \ldots \rightarrow S_{m}$ of $m$ disjoint subsets of $V$, such that $\cup_{i=1}^{m} S_{i}=V$, an ordered coloring of $G$ implied by the sequence $S_{1} \rightarrow S_{2} \rightarrow \ldots \rightarrow S_{k}$ is one in which the vertices of $S_{i}$ are assigned colors from $\left(\sum_{j=1}^{i-1}\left|S_{j}\right|\right)+1$ to $\sum_{j=1}^{i}\left|S_{j}\right|, i=1,2, \ldots, m$.

\subsection{The (2,n)-Differential Coloring Problem for Bipartite Graphs}

In this subsection, we consider the class of bipartite graphs. It is not difficult to see that the complement of a complete bipartite graphs is disconnected. Therefore, such a graph is not $(2, n)$-differential colorable. In the following, we prove that the reverse also holds.

Theorem 1. A bipartite graph admits a $(2, n)$-differential coloring if and only if it is not a complete bipartite graph.

Proof. Let $G=(V, E)$ be an $n$-vertex bipartite graph, with $V=V_{1} \cup V_{2}, V_{1} \cap$ $V_{2}=\emptyset$ and $E \subseteq V_{1} \times V_{2}$. If $G$ is a complete bipartite graph, then its complement is disconnected. Therefore, $G$ does not admit a $(2, n)$-differential coloring. Now, assume that $G$ is not a complete bipartite graph. Then, there exist at least two vertices, say $u \in V_{1}$ and $v \in V_{2}$, that are not adjacent, i.e., $(u, v) \notin E$. Consider the ordered coloring of $G$ implied by the sequence $V_{1} \backslash\{u\} \rightarrow\{u\} \rightarrow\{v\} \rightarrow V_{2} \backslash\{v\}$. As $u$ and $v$ are not adjacent, it follows that the color difference between any two vertices of $G$ is at least two. Hence, $G$ admits a $(2, n)$-differential coloring.

\subsection{The (2,n)-Differential Coloring Problem for Outerplanar Graphs}

In order to characterize which outerplanar graphs admit $(2, n)$-differential colorings, we follow an approach that consists of two main steps: First, we prove a special property of any 3-coloring of an outerplanar graph that does not contain as subgraph $K_{1, n-1}$, where $n \geq 6$ is the number of the graph's vertices. In a second step, we use this property to prove that such a graph always admits a $(2, n)$-differential coloring and vice versa.

Lemma 4. An outerplanar graph with $n \geq 6$ vertices, that does not contain $K_{1, n-1}$ as a subgraph, admits a 3-coloring, in which each color set contains at least 2 vertices.

Proof. Let $G=(V, E)$ be an outerplanar graph with $n \geq 6$ vertices, that does not contain $K_{1, n-1}$ as a subgraph. As $G$ is outerplanar, it admits a 3-coloring [23]. Let $C_{i} \subseteq V$ be the set of vertices of $G$ with color $i$ and $c_{i}$ be the number of vertices with color $i$, that is $c_{i}=\left|C_{i}\right|$, for $i=1,2,3$. Without loss of generality let $c_{1} \leq c_{2} \leq c_{3}$. We further assume that each color set contains at least one vertex, that is $c_{i} \geq 1$, $i=1,2,3$. If there is no set with less than 2 vertices, then the lemma clearly holds. Otherwise, we distinguish three cases:

C.1: $c_{1}=c_{2}=1$ and $c_{3} \geq 4$. Without loss of generality assume that $C_{1}=\{a\}$ and $C_{2}=\{b\}$. As $G$ is outerplanar, vertices $a$ and $b$ can have at most 2 common neighbors. On the other hand, since $G$ has at least 6 vertices, there exists at least one vertex, say $c \in C_{3}$, which is not a common neighbor of $a$ and $b$. Without loss of generality assume that $(b, c) \notin E$. Then, vertex $c$ can be colored with color 2. Therefore, we derive a new 3 -coloring of $G$ for which we have that $c_{1}=1$, $c_{2}=2$ and $c_{3} \geq 3$. 
C.2: $c_{1}=1, c_{2}=2$ and $c_{3} \geq 3$. Without loss of generality assume that $C_{1}=\{a\}$ and $C_{2}=\left\{b, b^{\prime}\right\}$. First, consider the case where there exists at least one vertex, say $c \in C_{3}$, which is not a neighbor of vertex $a$. In this case, vertex $c$ can be colored with color 1 and a new 3 -coloring of $G$ is derived with $c_{1}=c_{2}=2$ and $c_{3} \geq 3$, as desired. Now consider the more interesting case, where vertex $a$ is a neighbor of all vertices of $C_{3}$. As $G$ does not contain $K_{1, n-1}$ as a subgraph, either vertex $b$ or vertex $b^{\prime}$ is not a neighbor of vertex $a$. Without loss of generality let that vertex be $b$, that is $(a, b) \notin E$. As $G$ is outerplanar, vertices $a$ and $b^{\prime}$ can have at most 2 common neighbors. Since $G$ has at least 6 vertices and vertex $a$ is a neighbor of all vertices of $C_{3}$, there exist at least one vertex, say $c \in C_{3}$, which is not adjacent to vertex $b^{\prime}$, that is $\left(b^{\prime}, c\right) \notin E$. Therefore, we can color vertex $c$ with color 2 and vertex $b$ with color 1 and derive a new 3-coloring of $G$ for which we have that $c_{1}=c_{2}=2$ and $c_{3} \geq 2$, as desired.

C.3: $c_{1}=1, c_{2} \geq 3$ and $c_{3} \geq 3$. Without loss of generality assume that $C_{1}=\{a\}$. Then, there exists at least one vertex, say $c \in C_{2} \cup C_{3}$, which is not a neighbor of vertex $a$. In this case, vertex $c$ can be colored with color 1 and a new 3 -coloring of $G$ is derived with $c_{1}=c_{2}=2$ and $c_{3} \geq 3$, as desired.

From the above case analysis, it follows that indeed any outerplanar graph with $n \geq 6$ vertices, that does not contain $K_{1, n-1}$ as a subgraph, admits a 3 -coloring, in which each color set contains at least 2 vertices.

Lemma 5. Let $G=(V, E)$ be an outerplanar graph and let $V^{\prime}$ and $V^{\prime \prime}$ be two disjoint subsets of $V$, such that $\left|V^{\prime}\right| \geq 2$ and $\left|V^{\prime \prime}\right| \geq 3$. Then, there exist two vertices $u \in V^{\prime}$ and $v \in V^{\prime \prime}$, such that $(u, v) \notin E$.

Proof. The lemma follows from the fact that an outerplanar graph does not contain $K_{2,3}$ as a subgraph [24].

Theorem 2. An outerplanar graph with $n \geq 8$ vertices has $(2, n)$-differential coloring if and only if it does not contain $K_{1, n-1}$ as subgraph.

Proof. Let $G=(V, E)$ be an outerplanar graph with $n \geq 8$ vertices. If $G$ contains $K_{1, n-1}$ as subgraph, then the complement $G^{c}$ of $G$ is disconnected. Therefore, $G$ does not admit a $(2, n)$-differential coloring. Now, assume that $G$ does not contain $K_{1, n-1}$ as subgraph. By Lemma 4 it follows that $G$ admits a 3 -coloring, in which each color set contains at least two vertices. Let $C_{i} \subseteq V$ be the set of vertices with color $i$ and $c_{i}=\left|C_{i}\right|$, for $i=1,2,3$, such that $2 \leq c_{1} \leq c_{2} \leq c_{3}$. We distinguish the following cases:

C.1: $c_{1}=2, c_{2}=2$ and $c_{3} \geq 4$. Since $\left|C_{1}\right|=2$ and $\left|C_{3}\right| \geq 4$, by Lemma 5 it follows that there exist two vertices $a \in C_{1}$ and $c \in C_{3}$, such that $(a, c) \notin E$. Similarly, since $\left|C_{2}\right|=2$ and $\left|C_{3} \backslash\{c\}\right| \geq 3$, by Lemma 5 it follows that there exist two vertices $b \in C_{2}$ and $c^{\prime} \in C_{3}$, such that $c \neq c^{\prime}$ and $\left(b, c^{\prime}\right) \notin E$; see Fig. $2 \mathrm{a} 2 \mathrm{~b}$

C.2: $c_{1} \geq 2, c_{2} \geq 3$ and $c_{3} \geq 3$. Since $\left|C_{1}\right|=2$ and $\left|C_{3}\right| \geq 3$, by Lemma 5 it follows that there exist two vertices $a \in C_{1}$ and $c \in C_{3}$, such that $(a, c) \notin E$. Similarly, 


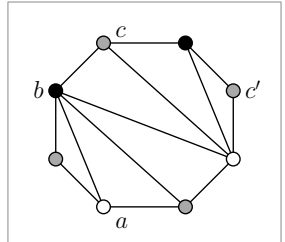

(a)

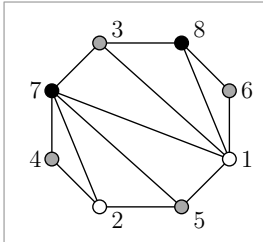

(b)

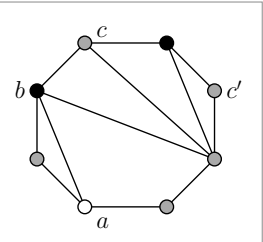

(c)

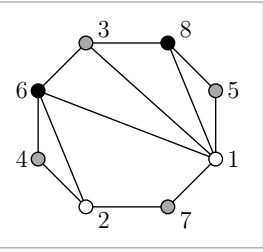

(d)

Figure 2: (a) An outerplanar graph colored with 3 colors, white, black and gray (Case 1 of Thm. 2), and, (b) its $(2, n)$-differential coloring. (c) Another outerplanar graph also colored with 3 colors, white, black and gray (Case 2 of Thm. 2), and, (d) its $(2, n)$-differential coloring.

since $\left|C_{2}\right| \geq 3$ and $\left|C_{3} \backslash\{c\}\right| \geq 2$, by Lemma 5 it follows that there exist two vertices $b \in C_{2}$ and $c^{\prime} \in C_{3}$, such that $c \neq c^{\prime}$ and $\left(b, c^{\prime}\right) \notin E$; see Fig. 2c $2 \mathrm{~d}$.

For both cases, consider the ordered coloring implied by the sequence $C_{1} \backslash\{a\} \rightarrow$ $\{a\} \rightarrow\{c\} \rightarrow C_{3} \backslash\left\{c, c^{\prime}\right\} \rightarrow\left\{c^{\prime}\right\} \rightarrow\{b\} \rightarrow C_{2} \backslash\{b\}$. As $(a, c) \notin E$ and $\left(b, c^{\prime}\right) \notin E$, it follows that the color difference between any two vertices of $G$ is at least two. Hence, $G$ admits a $(2, n)$-differential coloring.

Remark. Using the ILP formulation of Section 5, it can be shown that Theorem 2 holds for all outerplanar graphs with $n=7$ vertices. For $n \leq 6$ there exists in total five graphs for which Theorem 2 does not hold (see Figure 3 ).

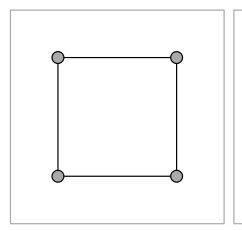

(a) $n=4$

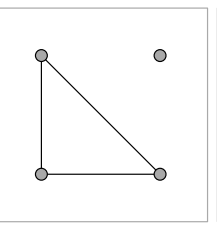

(b) $n=4$

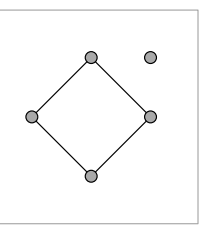

(c) $n=5$

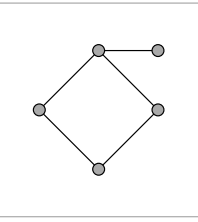

(d) $n=5$

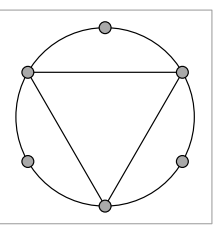

(e) $n=6$

Figure 3: Different graphs with $n \leq 6$ vertices which do not contain $K_{1, n-1}$ as subgraph but do not admit $(2, n)$-differential colorings.

In the particular case that the outerplanar graph is biconnected, we present an interesting property (that we will use in Section 3.3 regarding the hamiltonicity of the complement of a biconnected outerplanar graph on $n \geq 6$ vertices. However, before we proceed with the detailed proof of this property, we need first to introduce some necessary terminology. The weak-dual of a plane graph is the subgraph of its dual induced by neglecting the face-vertex corresponding to its unbounded face. A maximal outerplanar graph is called outer zig-zag, if its weak-dual is a path.

Lemma 6. Let $G=(V, E)$ be biconnected outerplanar on $n \geq 6$ vertices that (i) does not contain $K_{1, n-1}$ as a subgraph, and (ii) is not isomorphic to the one of Figure $3 e$ Then, $G^{c}$ contains a Hamiltonian path whose end-points are not consecutive on the outerface of $G$. 
Proof. We first augment $G$ by adding as many edges as possible without deviating outerplanarity and without introducing $K_{1, n-1}$ or the graph of Figure 3e, if $n=6$. If $n \geq 7$, then the resulting graph is always maximal outerplanar. In the case where $n=6$, the augmentation will result to a graph that is either isomorphic to an outer zigzag on 6 vertices or to the graph of Figure 3e without one chord. So, in the following we will assume w.l.o.g. that $G$ is the result of the aforementioned augmentation.

We will prove the lemma by induction on the number $n$ of vertices of $G$. The base of the induction corresponds to the case where $n=6$. In this particular case, it is not difficult to the check that the complements of the two graphs described above both contain Hamiltonian paths whose end-points are not consecutive on their outerfaces.

For the inductive step, consider a maximal outerplanar graph $G$ with $n>6$ vertices and assume that all outerplanar graphs with $n-1$ vertices that do not contain $K_{1, n-2}$ as subgraph, and are not isomorphic to the graph of Figure 3e contain Hamiltonian paths whose end-points are not consecutive on their outerfaces. Since $G$ is outerplanar, it contains at least two vertices, say $v$ and $v^{\prime}$, of degree at most two. If both $G-v$ and $G-v^{\prime}$ contain $K_{1, n-2}$, then it immediately follows that $G$ contains $K_{1, n-1}$ as a subgraph, a contradiction. So, we may assume w.l.o.g. that $G-v$ does not contain $K_{1, n-2}$ as a subgraph. We distinguish two cases: (C.1) $G-v$ is isomorphic to the graph of Figure 3e, and (C.2) $G-v$ is not isomorphic to the graph of Figure 3e Clearly in case C $1, G$ has exactly seven vertices. Let $u$ be the vertex of degree two in $G$ which is not incident to $v$ and has a common neighbour with $v$ in $G$. Then, $G-u$ is an outer zig-zag on 6 vertices. In case C2, graph $G-v$ is biconnected and does not contain $K_{1, n-2}$ as a subgraph.

It follows that in both cases there is always a vertex $w$ of degree at most two in $G$, such that $G-w$ is biconnected and satisfies the requirements of the Theorem (in case C $1 w$ is $u$, while in case $\mathrm{C} 2 w$ is $v)$. By the induction hypothesis, $(G-w)^{c}$ has a Hamiltonian path, say $P$, whose endpoints are not consecutive on the outerface of $G-w$ and therefore in $G$. Since $G$ has at least 7 vertices and the degree of $v$ is at most two in $G$, there exist two consecutive internal vertices of $P$, say $w_{1}$ and $w_{2}$, such that $\left(w, w_{1}\right) \notin E$ and $\left(w, w_{2}\right) \notin E$. This implies that $G^{c}$ has also a Hamiltonian path whose end-points are not consecutive on the outerface of $G$ and the proof is complete.

Lemma 7. Let $G=(V, E)$ be biconnected outerplanar on $n \geq 6$ with $\operatorname{diam}(G) \geq 3$. Then, $G^{c}$ contains a Hamiltonian path whose end-points are not consecutive on the outerface of $G$.

Proof. Since $\operatorname{diam}(G) \geq 3$, graph $G$ cannot contain $K_{1, n-1}$ as a subgraph. In addition, $G$ cannot be isomorphic to the graph of Figure $3 \mathrm{e}$, as the later graph has diameter two. Hence, by Theorem 6 graph $G$ contains a Hamiltonian path whose end-points are not consecutive on the outerface of $G$.

\subsection{The (2,n)-Differential Coloring Problem for Planar Graphs}

In this subsection, we provide a complete characterization for planar graphs admitting $(2, n)$-differential colorings. First, we give a simple and quite intuitive proof of this characterization, which however holds for planar graphs with $n \geq 36$ vertices. Inspired from this proof and using more complicated arguments we prove that our 
characterization can be extended to planar graphs with $n \geq 12$ vertices. As in the case of outerplanar graphs, our first proof consists of two main steps: First, we prove a special property of any 4-coloring of a planar graph that does not contain as subgraphs $K_{1,1, n-3}, K_{1, n-1}$ and $K_{2, n-2}$, where $n \geq 36$ is the number of the graph's vertices. Then, we employ this property to prove that such a graph always admits a $(2, n)$-differential coloring.

Lemma 8. A planar graph with $n \geq 36$ vertices, that does not contain as subgraphs $K_{1,1, n-3}, K_{1, n-1}$ and $K_{2, n-2}$, admits a 4 -coloring, in which two color sets contain at least 2 vertices and the remaining two at least 5 vertices.

Proof. Let $G=(V, E)$ be a planar graph with $n \geq 36$ vertices, that does not contain as subgraphs $K_{1,1, n-3}, K_{1, n-1}$ and $K_{2, n-2}$. As $G$ is planar, it admits a 4-coloring [25]. Let $C_{i} \subseteq V$ be the set of vertices of $G$ with color $i$ and $c_{i}$ be the number of vertices with color $i$, that is $c_{i}=\left|C_{i}\right|$, for $i=1,2,3,4$. Without loss of generality let $c_{1} \leq$ $c_{2} \leq c_{3} \leq c_{4}$. We further assume that each color set contains at least one vertex, that is $c_{i} \geq 1, i=1,2,3,4$. We distinguish the following cases:

C.1: $c_{1}=1, c_{2}=1$ and $c_{3} \leq 7$. Since $G$ has at least 36 vertices, it follows that $c_{4} \geq$ 27 . Observe that a vertex of $C_{4}$ cannot be assigned a color other than 4 if and only if it is adjacent to at least one vertex in $C_{1}$, one vertex in $C_{2}$ and one vertex in $C_{3}$. Let $C_{4}^{*} \subseteq C_{4}$ be the set of such vertices and $c_{4}^{*}=\left|C_{4}^{*}\right|$. We claim that $c_{4}^{*} \leq 14$. To prove the claim, we construct an auxiliary bipartite graph $G_{\text {aux }}=\left(V_{\text {aux }}, E_{\text {aux }}\right)$ where $V_{\text {aux }}=C_{1} \cup C_{2} \cup C_{3} \cup C_{4}^{*}$ and $E_{\text {aux }}=E \cap\left(C_{4}^{*} \times\left(C_{1} \cup C_{2} \cup C_{3}\right)\right)$. Clearly, $G_{\text {aux }}$ is planar, since it is subgraph of $G$. Since all vertices of $C_{4}^{*}$ have degree at least 3 , it holds that $3 c_{4}^{*} \leq\left|E_{\text {aux }}\right|$. On the other hand, it is known that a planar bipartite graph on $n \geq 3$ vertices cannot have more that $2 n-4$ edges [26]. Therefore, $\left|E_{\text {aux }}\right| \leq 2\left(c_{1}+c_{2}+c_{3}+c_{4}^{*}\right)-4$, which implies that $3 c_{4}^{*} \leq 2\left(c_{1}+c_{2}+c_{3}+c_{4}^{*}\right)-4$. As $c_{1}+c_{2}+c_{3} \leq 9$, it follows that our claim indeed holds. A a consequence, we can change the color of several vertices initially in $C_{4}$ and obtain a new 4-coloring of $G$ in which $C_{4}$ has exactly 14 vertices. This implies that the number of vertices in $C_{1}, C_{2}$ and $C_{3}$ is at least 22 or equivalently that one out of $C_{1}, C_{2}$ and $C_{3}$ must contain strictly more that 7 vertices, say $C_{3}$. So, in the new coloring it holds that $c_{1} \geq 1, c_{2} \geq 1$ and $c_{3} \geq 8$; a case that is covered in the following.

C.2: $c_{1}=1, c_{2}=1$ and $c_{3} \geq 8$. Assume without loss of generality that $C_{1}=\{a\}$ and $C_{2}=\{b\}$. Since $G$ does not contain $K_{2, n-2}$ as subgraph, there exists at least one vertex, say $c \in C_{3} \cup C_{4}$, which is not connected to both $a$ and $b$; say, $(b, c) \notin E$. Then, vertex $c$ can be safely colored with color 2 . In the new coloring, it holds that $c_{1}=1, c_{2}=2$ and $c_{3} \geq 7$; refer to Case 4

C.3: $c_{1}=1, c_{2}=2$ and $c_{3} \leq 6$. Since $G$ has at least 36 vertices, it follows that $c_{4} \geq 27$. Now, observe that $c_{1}+c_{2}+c_{3} \leq 9$. Hence, following similar arguments as in Case 1 , we can prove that there is a 4 -coloring of $G$ in which $C_{4}$ has exactly 14 vertices. So, again the number of vertices in $C_{1}, C_{2}$ and $C_{3}$ is at least 22 and consequently at least one out of $C_{1}, C_{2}$ and $C_{3}$ must contain strictly more that 7 
vertices, say $C_{3}$. In the new coloring, it holds that $c_{1} \geq 1, c_{2} \geq 2$ and $c_{3} \geq 8$; refer to Case 4.

C.4: $c_{1}=1, c_{2}=2$ and $c_{3} \geq 7$. Assume without loss of generality that $C_{1}=\{a\}$ and $C_{2}=\left\{b, b^{\prime}\right\}$. First, consider the case where there exists at least one vertex, say $c \in C_{3} \cup C_{4}$, which is not a neighbor of vertex $a$. In this particular case, vertex $c$ can be colored with color 1 and a new 4-coloring of $G$ is derived for which it holds that $c_{1}=2, c_{2}=2$ and $c_{3} \geq 6$, as desired. Now, consider the more interesting case, where vertex $a$ is a neighbor of all vertices in $C_{3} \cup C_{4}$. As $G$ does not contain $K_{1, n-1}$ as subgraph, vertex $a$ is not a neighbor of vertex $b$ or vertex $b^{\prime}$ or both. In the latter case, vertex $a$ can be colored with color 2 . Therefore, if we select two arbitrary vertices of $C_{3}$ and color them with color 1 , we derive a new 4-coloring of $G$ for which it holds that $c_{1}=2, c_{2}=2$ and $c_{3} \geq 5$, as desired. On the other hand, if vertex $a$ is a neighbor of exactly one of $b$ and $b^{\prime}$, say without loss of generality $b$, that is $(a, b) \in E$ and $\left(a, b^{\prime}\right) \notin E$, then there exists a vertex $c \in C_{3} \cup C_{4}$ such that $(b, c) \notin E$; as otherwise the complete tripartite graph formed by vertex $a$, vertex $b$ and $C_{3} \cup C_{4}$ forms a $K_{1,1, n-3}$. So, if we color vertex $b^{\prime}$ with color 1 and vertex $c$ with color 2 , we obtain a new coloring of $G$ for which it holds that $c_{1}=2, c_{2}=2$ and $c_{3} \geq 6$, as desired.

C.5: $c_{1}=1, c_{2} \geq 3$ and $c_{3} \geq 3$. Assume without loss of generality that $C_{1}=\{a\}$. As $G$ does not contain $K_{1, n-1}$ as subgraph, there is a vertex $a^{\prime} \in C_{2} \cup C_{3} \cup C_{4}$ which is not a neighbor of $a$. Hence, $a^{\prime}$ can be colored with color 1 . In the new coloring, it holds that $c_{1} \geq 2, c_{2} \geq 2, c_{3} \geq 2$; refer to Case 6

C.6: $c_{1} \geq 2, c_{2} \geq 2$ and $c_{3} \leq 4$. Since $G$ has at least 36 vertices, it follows that $c_{4} \geq 24$. Since $c_{1}+c_{2}+c_{3} \leq 12$, following similar arguments as in Case 1 we can prove that there is a 4 -coloring of $G$ in which $C_{4}$ has exactly 20 vertices. So, the number of vertices in $C_{1}, C_{2}$ and $C_{3}$ is at least 16 and consequently at least one out of $C_{1}, C_{2}$ and $C_{3}$ must contain strictly more that 5 vertices, say $C_{3}$. In the new coloring, it holds that $c_{1} \geq 2, c_{2} \geq 2$ and $c_{3} \geq 6$, as desired.

From the above case analysis, it follows that $G$ has a 4-coloring, in which two color sets contain at least 2 vertices and the remaining two at least 5 vertices.

Lemma 9. Let $G=(V, E)$ be a planar graph and let $V^{\prime}$ and $V^{\prime \prime}$ be two disjoint subsets of $V$, such that $\left|V^{\prime}\right| \geq 3$ and $\left|V^{\prime \prime}\right| \geq 3$. Then, there exist two vertices $u \in V^{\prime}$ and $v \in V^{\prime \prime}$, such that $(u, v) \notin E$.

Proof. The lemma follows directly from the fact that a planar graph does not contain $K_{3,3}$ as subgraph [27].

Theorem 3. A planar graph with $n \geq 36$ vertices has a $(2, n)$-differential coloring if and only if it does not contain as subgraphs $K_{1,1, n-3}, K_{1, n-1}$ and $K_{2, n-2}$.

Proof. Let $G=(V, E)$ be an $n$-vertex planar graph, with $n \geq 36$. If $G$ contains $K_{l, n-l}$ as subgraph, then the complement $G^{c}$ of $G$ is disconnected, $l=1,2 \ldots,\lceil n / 2\rceil$. Therefore, $G$ has no $(2, n)$-differential coloring. Note, however, that since $G$ is planar, 


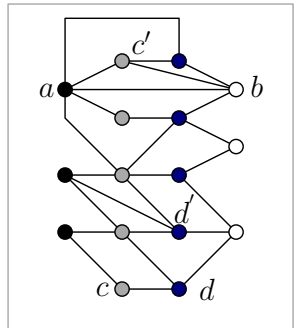

(a)

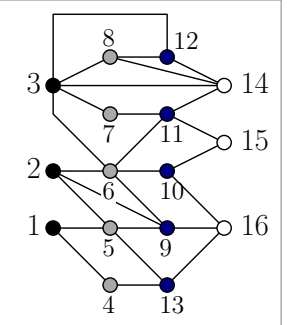

(b)

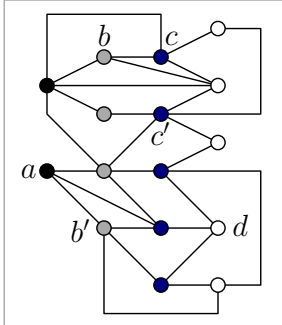

(c)

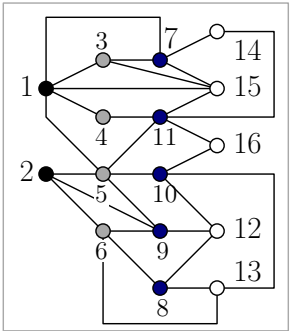

(d)

Figure 4: (a) A planar graph colored with 4 colors, black $\left(C_{1}\right)$, white $\left(C_{2}\right)$, gray $\left(C_{3}\right)$ and blue $\left(C_{4}\right)$; Case 1 of Thm. 3 and, (b) its $(2, n)$-differential coloring. (c) Another planar graph also colored with 4 colors, black, gray, blue and white; Case 2 of Thm. 3 , and, (d) its $(2, n)$-differential coloring.

it cannot contain $K_{l, n-l}, l=3,4 \ldots,\lceil n / 2\rceil$, as subgraph. On the other hand, if $G$ contains $K_{1,1, n-3}$ as subgraph, then $G^{c}$ has two vertices of degree at most one, whose neighborhoods are subsets of the same vertex (as a singleton set). Hence, $G^{c}$ is not Hamiltonian and as a consequence $G$ has no $(2, n)$-differential coloring, as well.

Now, assume that $G$ does not contain as subgraphs $K_{1,1, n-3}, K_{1, n-1}$ and $K_{2, n-2}$. By Lemma 8 , it follows that $G$ admits a 4-coloring, in which two color sets contain at least 2 vertices and the remaining two at least 5 vertices. The main strategy of our approach is to compute ordered colorings implied by sequences of vertices such that adjacent vertices in the sequence are not connected by an edge. Let $C_{i} \subseteq V$ be the set of vertices with color $i$ and $c_{i}=\left|C_{i}\right|$, for $i=1,2,3,4$, such that $c_{1} \leq c_{2} \leq c_{3} \leq c_{4}$, $c_{1}, c_{2} \geq 2$ and $c_{3}, c_{4} \geq 5$. We distinguish the following cases:

C.1: $c_{1} \geq 3, c_{2} \geq 3, c_{3} \geq 5$ and $c_{4} \geq 5$. Since $\left|C_{1}\right| \geq 3$ and $\left|C_{3}\right| \geq 5$, by Lemma 9 it follows that there exist two vertices $a \in C_{1}$ and $c \in C_{3}$, such that $(a, c) \notin E$. Similarly, since $\left|C_{2}\right| \geq 3$ and $\left|C_{4}\right| \geq 5$, by Lemma 9 it follows that there exist two vertices $b \in C_{2}$ and $d \in C_{4}$, such that $(b, d) \notin E$. Finally, since $\left|C_{3} \backslash\{c\}\right| \geq 4$ and $\left|C_{4} \backslash\{d\}\right| \geq 4$, by Lemma 9 it follows that there exist two vertices $c^{\prime} \in C_{3}$ and $d^{\prime} \in C_{4}$, such that $c \neq c^{\prime}, d \neq d^{\prime}$ and $\left(c^{\prime}, d^{\prime}\right) \notin E$. Now consider the ordered coloring implied by the sequence $C_{1} \backslash\{a\} \rightarrow\{a\} \rightarrow$ $\{c\} \rightarrow C_{3} \backslash\left\{c, c^{\prime}\right\} \rightarrow\left\{c^{\prime}\right\} \rightarrow\left\{d^{\prime}\right\} \rightarrow C_{4} \backslash\left\{d, d^{\prime}\right\} \rightarrow\{d\} \rightarrow\{b\} \rightarrow C_{2} \backslash\{b\}$. As $(a, c) \notin E,(b, d) \notin E$ and $\left(c^{\prime}, d^{\prime}\right) \notin E$, it follows that the color difference between any two vertices of $\mathrm{G}$ is at least two. Hence, $G$ has a $(2, n)$-differential coloring.

C.2: $c_{1}=2, c_{2} \geq 4, c_{3} \geq 5$ and $c_{4} \geq 5$. Since $G$ does not contain $K_{2, n-2}$ as subgraph, there exist two vertices, say $a \in C_{1}$ and $b \in C_{2} \cup C_{3} \cup C_{4}$ such that $(a, b) \notin E$. Assume without loss of generality that $b \in C_{2}$. Since $\left|C_{2} \backslash\{b\}\right| \geq 3$ and $\left|C_{3}\right| \geq 5$, by Lemma 9 it follows that there exist two vertices $b^{\prime} \in C_{2}$ and $c \in C_{3}$, such that $b^{\prime} \neq b$ and $\left(b^{\prime}, c\right) \notin E$. Similarly, since $\left|C_{3} \backslash\{c\}\right| \geq 4$ and $\left|C_{4}\right| \geq 5$, by Lemma 9 it follows that there exist two vertices $c^{\prime} \in C_{3}$ and $d \in C_{4}$, such that $c^{\prime} \neq c$ and $\left(c^{\prime}, d\right) \notin E$. As in the previous case, the ordered 
coloring implied by the sequence $C_{1} \backslash\{a\} \rightarrow\{b\} \rightarrow C_{2} \backslash\{b\} \rightarrow\left\{b^{\prime}\right\} \rightarrow\{c\} \rightarrow$ $C_{3} \backslash\left\{c, c^{\prime}\right\} \rightarrow\left\{c^{\prime}\right\} \rightarrow\{d\} \rightarrow C_{4} \backslash\{d\}$ guarantees that $G$ has a $(2, n)$-differential coloring.

C.3: $c_{1}=2, c_{2}=3, c_{3} \geq 5$ and $c_{4} \geq 5$. Assume without loss of generality that $C_{1}=\left\{a, a^{\prime}\right\}$ and $C_{2}=\left\{b, b^{\prime}, b^{\prime \prime}\right\}$. We distinguish two sub-cases:

C.3.1: The subgraph of $G$ induced by $C_{1} \cup C_{2}$ is $K_{2,3}$, that is $C_{1} \times C_{2} \subseteq E$. Since $G$ does not contain $K_{2, n-2}$ as subgraph, there exists at least one vertex of $C_{1}$, say vertex $a$, that is not a neighbor of a vertex of $C_{3} \cup C_{4}$, say vertex $c \in C_{3}$, that is $(a, c) \notin E$. Since $\left|C_{2}\right|=3$ and $\left|C_{4}\right| \geq 5$, by Lemma 9 it follows that there exist a vertex of $C_{2}$, say vertex $b$, and a vertex of $C_{4}$, say vertex $d$, that are not adjacent, that is $(b, d) \notin E$. Similarly, since $\left|C_{3} \backslash\{c\}\right| \geq 4$ and $\left|C_{4} \backslash\{d\}\right| \geq 4$, by Lemma 9 it follows that there exist two vertices $c^{\prime} \in C_{3}$ and $d^{\prime} \in C_{4}$, such that $c \neq c^{\prime}$, $d \neq d^{\prime}$ and $\left(c^{\prime}, d^{\prime}\right) \notin E$. Thus in the ordered coloring implied by the sequence $C_{1} \backslash\{a\} \rightarrow\{a\} \rightarrow\{c\} \rightarrow C_{3} \backslash\left\{c, c^{\prime}\right\} \rightarrow\left\{c^{\prime}\right\} \rightarrow\left\{d^{\prime}\right\} \rightarrow$ $C_{4} \backslash\left\{d, d^{\prime}\right\} \rightarrow\{d\} \rightarrow\{b\} \rightarrow C_{2} \backslash\{b\}$, it holds that the color difference between any two vertices of $G$ is greater or equal to two. Hence, $G$ admits a $(2, n)$-differential coloring.

C.3.2: The subgraph of $G$ induced by $C_{1} \cup C_{2}$ is not $K_{2,3}$. Assume without loss of generality that $(a, b) \notin E$. Since $\left|C_{3}\right| \geq 5$ and $\left|C_{4}\right| \geq 5$, by Lemma 9 it follows that there exist a vertex of $C_{3}$, say vertex $c$, and a vertex of $C_{4}$, say vertex $d$, that are not adjacent, that is $(c, d) \notin E$. Similarly, since $\left|C_{3} \backslash\{c\}\right| \geq 4$ and $\left|\left\{a^{\prime}, b^{\prime}, b^{\prime \prime}\right\}\right|=3$, by Lemma 9 it follows that there exist a vertex of $C_{3} \backslash\{c\}$, say vertex $c^{\prime}$, and a vertex of $\left\{a^{\prime}, b^{\prime}, b^{\prime \prime}\right\}$, say vertex $x$, that are not adjacent, that is $\left(x, c^{\prime}\right) \notin E$. First consider the case where $x=a^{\prime}$. In this case, the ordered coloring implied by the sequence $\left\{b^{\prime}\right\} \rightarrow\left\{b^{\prime \prime}\right\} \rightarrow\{b\} \rightarrow\{a\} \rightarrow\left\{a^{\prime}\right\} \rightarrow\left\{c^{\prime}\right\} \rightarrow C_{3} \backslash\left\{c, c^{\prime}\right\} \rightarrow\{c\} \rightarrow$ $\{d\} \rightarrow C_{4} \backslash\{d\}$ guarantees that $G$ has a $(2, n)$-differential coloring. Now consider the case, where $x \in\left\{b^{\prime}, b^{\prime}\right\}$. As both cases are symmetric, we assume without loss of generality that $x=b^{\prime}$. In this case, the ordered coloring implied by the sequence $\left\{a^{\prime}\right\} \rightarrow\{a\} \rightarrow\{b\} \rightarrow\left\{b^{\prime \prime}\right\} \rightarrow\left\{b^{\prime}\right\} \rightarrow$ $\left\{c^{\prime}\right\} \rightarrow C_{3} \backslash\left\{c, c^{\prime}\right\} \rightarrow\{c\} \rightarrow\{d\} \rightarrow C_{4} \backslash\{d\}$ guarantees that $G$ has a $(2, n)$-differential coloring.

C.4: $c_{1}=2, c_{2}=2, c_{3} \geq 5$ and $c_{4} \geq 5$. Assume without loss of generality that $C_{1}=\left\{a, a^{\prime}\right\}$ and $C_{2}=\left\{b, b^{\prime}\right\}$. Again, we distinguish two sub-cases:

C.4.1: The subgraph of $G$ induced by $C_{1} \cup C_{2}$ is $K_{2,2}$, that is $C_{1} \times C_{2} \subseteq E$. Since $G$ does not contain $K_{2, n-2}$ as subgraph, there exists at least one vertex of $C_{1}$, say vertex $a$, that is not a neighbor of a vertex of $C_{3} \cup C_{4}$, say vertex $c \in C_{3}$, that is $(a, c) \notin E$. Similarly, there exists a vertex of $C_{2}$, say vertex $b$, that is not a neighbor of a vertex of $C_{3} \cup C_{4}$, say vertex $w$, that is $(b, w) \notin E$.

First, consider the case where the subgraph of $G$ induced by $C_{2} \cup C_{4}$ is not $K_{2, c_{4}}$ that is $w \in C_{4}$. Since $\left|C_{3} \backslash\{c\}\right| \geq 4$ and $\left|C_{4} \backslash\{w\}\right| \geq 4$, by 
Lemma 9 it follows that there exists a vertex of $C_{3}$, say $c^{\prime}$, and a vertex of $C_{4}$, say $d$, that are not adjacent, that is $c \neq c^{\prime}, w \neq d$ and $\left(c^{\prime}, d\right) \notin E$. In this case, the ordered coloring implied by the sequence $C_{1} \backslash\{a\} \rightarrow$ $\{a\} \rightarrow\{c\} \rightarrow C_{3} \backslash\left\{c, c^{\prime}\right\} \rightarrow\left\{c^{\prime}\right\} \rightarrow\{d\} \rightarrow C_{4} \backslash\{d, w\} \rightarrow\{w\} \rightarrow$ $\{b\} \rightarrow C_{2} \backslash\{b\}$ guarantees that $G$ has a $(2, n)$-differential coloring.

Now consider the more interesting case where the subgraph of $G$ induced by $C_{2} \cup C_{4}$ is $K_{2, c_{4}}$, that is $w \in C_{3}$. We distinguish two sub cases:

C.4.1.1: $w \neq c$. Since $\left|C_{3} \backslash\{c, w\}\right| \geq 3$ and $\left|C_{4}\right| \geq 5$, by Lemma 9 it follows that there exist vertices $p \in C_{3}$ and $q \in C_{4}$, that are not adjacent, that is $c \neq w \neq p$, and $(p, q) \notin E$. Similarly, since $\left|C_{3} \backslash\{p, w\}\right| \geq 3$ and $\left|C_{4} \backslash\{q\}\right| \geq 4$, by Lemma 9 it follows that there exist vertices $p^{\prime} \in C_{3}$ and $q^{\prime} \in C_{4}$, that are not adjacent, that is $p \neq w \neq p^{\prime}, q \neq q^{\prime}$ and $\left(p^{\prime}, q^{\prime}\right) \notin E$. If $p^{\prime} \neq c$, the ordered coloring implied by the sequence $C_{1} \backslash\{a\} \rightarrow$ $\{a\} \rightarrow\{c\} \rightarrow\left\{p^{\prime}\right\} \rightarrow\left\{q^{\prime}\right\} \rightarrow C_{4} \backslash\left\{q, q^{\prime}\right\} \rightarrow\{q\} \rightarrow\{p\} \rightarrow$ $C_{3} \backslash\left\{p, p^{\prime}, c, w\right\} \rightarrow\{w\} \rightarrow\{b\} \rightarrow C_{2} \backslash\{b\}$ guarantees that $G$ has a $(2, n)$-differential coloring. If $p^{\prime}=c$, the ordered coloring implied by the sequence $C_{1} \backslash\{a\} \rightarrow\{a\} \rightarrow\left\{p^{\prime}\right\} \rightarrow\left\{q^{\prime}\right\} \rightarrow$ $C_{4} \backslash\left\{q, q^{\prime}\right\} \rightarrow\{q\} \rightarrow\{p\} \rightarrow C_{3} \backslash\left\{p, p^{\prime}, w\right\} \rightarrow\{w\} \rightarrow\{b\} \rightarrow$ $C_{2} \backslash\{b\}$ guarantees that $G$ has a $(2, n)$-differential coloring.

C.4.1.2: $w=c$. Since the subgraph of $G$ induced by $C_{2} \cup C_{4}$ is $K_{2, c_{4}}$ and $\left|C_{2} \cup\{a\}\right|=3$ and $\left|C_{4}\right| \geq 4$, by Lemma 9 it follows that there exist a vertex of $C_{4}$, say $q$, not adjacent to vertex $a$, that is $(a, q) \notin E$. Similarly, since $\left|C_{3} \backslash\{c\}\right| \geq 4$ and $\left|C_{4} \backslash\{q\}\right| \geq 4$, by Lemma 9 it follows that there exist vertices $p^{\prime} C_{3}$ and $q^{\prime} \in C_{4}$, that are not adjacent, that is $q^{\prime} \neq q, p^{\prime} \neq c$ and $\left(p^{\prime}, q^{\prime}\right) \notin E$. Then the ordered coloring implied by the sequence $C_{1} \backslash\{a\} \rightarrow\{a\} \rightarrow$ $\{q\} \rightarrow C_{4} \backslash\left\{q, q^{\prime}\right\} \rightarrow\left\{q^{\prime}\right\} \rightarrow\left\{p^{\prime}\right\} \rightarrow C_{3} \backslash\left\{p^{\prime}, c\right\} \rightarrow\{c\} \rightarrow$ $\{b\} \rightarrow C_{2} \backslash\{b\}$ guarantees that $G$ is $(2, n)$-differential colorable.

C.4.2: The subgraph of $G$ induced by $C_{1} \cup C_{2}$ is not $K_{2,2}$. Assume without loss of generality that $(a, b) \notin E$.

First, consider the case where the subgraph of $G$ induced by $\left\{a^{\prime}, b^{\prime}\right\} \cup$ $C_{3} \cup C_{4}$ is $K_{2, c_{3}+c_{4}}$ that is $\left\{a^{\prime}, b^{\prime}\right\} \times C_{3} \cup C_{4} \subseteq E$. Since $\left|\left\{a, a^{\prime}, b^{\prime}\right\}\right|=3$ and $\left|C_{3}\right| \geq 4$, by Lemma 9 it follows that there exists a vertex of $C_{3}$, say vertex $c$, not adjacent to vertex a, that is $(a, c) \notin E$. Similarly, since $\left|\left\{b, a^{\prime}, b^{\prime}\right\}\right|=3$ and $\left|C_{4}\right| \geq 4$, by Lemma 9 it follows that there exists a vertex of $C_{4}$, say vertex $d$, not adjacent to vertex b, that is $(b, d) \notin E$. Since $\left|C_{3} \backslash\{c\}\right| \geq 3$ and $\left|C_{4} \backslash\{d\}\right| \geq 4$, by Lemma 9 it follows that there exist a vertex of $C_{3}$, say vertex $c^{\prime}$, and a vertex of $C_{4}$, say vertex $d^{\prime}$, that are not adjacent, that is $c \neq c^{\prime}, d \neq d^{\prime}$ and $\left(c^{\prime}, d^{\prime}\right) \notin E$. Then the ordered coloring implied by the sequence $C_{1} \backslash\{a\} \rightarrow\{a\} \rightarrow\{c\} \rightarrow$ $C_{3} \backslash\left\{c, c^{\prime}\right\} \rightarrow\left\{c^{\prime}\right\} \rightarrow\left\{d^{\prime}\right\} \rightarrow C_{4} \backslash\left\{d, d^{\prime}\right\} \rightarrow\{d\} \rightarrow\{b\} \rightarrow C_{2} \backslash\{b\}$ guarantees that $G$ has a $(2, n)$-differential coloring.

Now we consider the case where the subgraph of $G$ induced by $\left\{a^{\prime}, b^{\prime}\right\} \cup$ $C_{3} \cup C_{4}$ is not $K_{2, c_{3}+c_{4}}$. There exist vertices $c \in C_{3} \cup C_{4}$ and $p \in\left\{a^{\prime}, b^{\prime}\right\}$, 
that are not adjacent, that is $(p, c) \notin E$. Assume without loss of generality $c \in C_{3}$. Since $\left|C_{3} \backslash\{c\}\right| \geq 3$ and $\left|C_{4}\right| \geq 4$, by Lemma 9 it follows that there exist vertices $c^{\prime} \in C_{3}$ and $d \in C_{4}$, that are not adjacent, that is $c \neq c^{\prime}$ and $\left(c^{\prime}, d^{\prime}\right) \notin E$. If $p=b^{\prime}$ the ordered coloring implied by the sequence $\left\{a^{\prime}\right\} \rightarrow\{a\} \rightarrow\{b\} \rightarrow\left\{b^{\prime}\right\} \rightarrow\{c\} \rightarrow C_{3} \backslash\left\{c, c^{\prime}\right\} \rightarrow\left\{c^{\prime}\right\} \rightarrow\left\{d^{\prime}\right\} \rightarrow$ $C_{4} \backslash\left\{d^{\prime}\right\}$ guarantees that $G$ has a $(2, n)$-differential coloring. If $p=a^{\prime}$ the ordered coloring implied by the sequence $\left\{b^{\prime}\right\} \rightarrow\{b\} \rightarrow\{a\} \rightarrow\left\{a^{\prime}\right\} \rightarrow$ $\{c\} \rightarrow C_{3} \backslash\left\{c, c^{\prime}\right\} \rightarrow\left\{c^{\prime}\right\} \rightarrow\left\{d^{\prime}\right\} \rightarrow C_{4} \backslash\left\{d^{\prime}\right\}$ guarantees that $G$ has a $(2, n)$-differential coloring.

From the above case analysis, it follows that $G$ is $(2, n)$-differential colorable.

As already stated, with more complicated arguments we are able to show that Theorem 3 holds for all planar graphs with $n \geq 12$ vertices. Recall that Theorem 3 guarantees the existence of a $(2, n)$-differential coloring for graphs with $n \geq 36$ vertices. The proof consists of two main parts: In the first part of the proof, we show that all planar graphs with $n \geq 12$ vertices that do not contain as subgraphs $K_{1,1, n-3}, K_{1, n-1}$ and $K_{2, n-2}$ admit 6 -colorings with the following two properties:

P.1 Each color class contains at least two vertices.

P.2 There exists a Hamiltonian path in the complement of the graph, which visits all vertices of the $i$-th colored class before visiting the vertices of the $(i+1)$-th colored class, $i=1, \ldots, 5$, such that the edges that connect vertices of consecutive color classes are independent.

We refer to a 6-coloring satisfying properties P.1 and P.2 as a good 6-coloring. In the second part of the proof, we show how a good 6 -coloring can be employed to show that any planar graph with $n \geq 12$ vertices that does not contain as subgraphs $K_{1,1, n-3}$, $K_{1, n-1}$ and $K_{2, n-2}$ admits $(2, n)$-differential coloring.

We use induction to prove that all planar graphs with $n \geq 12$ vertices that do not contain as subgraphs $K_{1,1, n-3}, K_{1, n-1}$ and $K_{2, n-2}$ admit good 6-colorings. The base of the induction corresponds to the case where $n=12$. To prove that all planar graphs with $n=12$ vertices that do not contain as subgraphs $K_{1,1,9}, K_{1,11}$ and $K_{2,10}$ admit good 6-colorings is a tedious task, which we postpone for the time being (as many different cases need to be considered; see Corollary 1 of Lemma 13 and instead we present a more intuitive computer-aided approach: First, we generated all planar (non-isomorphic to each other) triangulations with $n=12$ vertices (there are 7,595 of them) using plantri [28]. We used a simple integer linear program to test whether there is an assignment of the vertices of each of these graphs to one of the two available colors of each color class, such that both properties of a good 6-coloring hold (details follow). In total, we found 233 infeasible instances, all of which contained one of $K_{1,1,9}, K_{1,11}, K_{2,10}$ as a subgraph. From this we can conclude that the claim holds for all triangulations with $n=12$ vertices. Note that this implies that it also holds for all planar graphs with 12 vertices, as one can always augment a planar graph to maximal planar without introducing all forbitten subgraphs mentioned above.

For the sake of completeness, in the following we give an outline of the integer linear program above. For a vertex $u \in V$ of the input graph $G$ and a color $c$ of color 
set $C=\{1, \ldots, 6\}$, the boolean variable $x_{u, c}^{(k)}$ indicates whether $u$ is the $k$-th vertex with color $c$, where $k=1,2$. The first three constraints ensure that all vertices of $G$ are properly colored (with colors from 1 to 6 ). The last two constraints guarantee property P. 2 of a good 6-coloring.

$$
\begin{aligned}
& \text { maximize } O P T \\
& \text { subject to } \quad \sum_{u \in V} x_{u, c}^{(k)} \quad=1 \quad c \in C ; k \in\{1,2\} \\
& \sum_{c \in C} x_{u, c}^{(k)} \quad=1 \quad u \in V ; k \in\{1,2\} \\
& \sum_{k=1}^{2} x_{u, c}^{(k)}+x_{v, c}^{(k)} \leq 1 \quad \forall(u, v) \in E ; c \in C \\
& x_{u, c}^{(1)}+x_{u, c+1}^{(1)}+x_{v, c}^{(1)}+x_{v, c+1}^{(1)} \leq 1 \quad \forall(u, v) \in E ; c \in\{1,3,5\} \\
& x_{u, c}^{(2)}+x_{u, c+1}^{(2)}+x_{v, c}^{(2)}+x_{v, c+1}^{(2)} \leq 1 \quad \forall(u, v) \in E ; c \in\{2,4\}
\end{aligned}
$$

For the inductive step, assume that our claim holds for all planar graphs with a certain amount of vertices, say $n_{0} \geq 12$, and consider a planar graph, say $G$, with $n_{0}+1$ vertices that does not contain as subgraphs $K_{1,1, n_{0}-2}, K_{1, n_{0}}$ and $K_{2, n_{0}-1}$. By Euler's formula, it follows that $G$ contains at least four vertices, say $v_{1}, v_{2}, v_{3}$ and $v_{4}$, of degree less than 5 . First, consider the case where at least one of the graphs $G-v_{1}$, $G-v_{2}, G-v_{3}$ and $G-v_{4}$ contains neither $K_{1,1, n_{0}-3}$ nor $K_{1, n_{0}-1}$ nor $K_{2, n_{0}-2}$ as subgraphs, say w.l.o.g. $G-v_{1}$. By the inductive hypothesis, it follows that $G-v_{1}$ admits a good 6-coloring. Since $\operatorname{deg}\left(v_{1}\right) \leq 5$, it follows that $v_{1}$ is incident to at most 5 vertices of potentially different colors in $G-v_{1}$. Hence, we can color $v_{1}$ with the sixth available color, say $c$. Observe that in the coloring of $G$ each color class will still have at least two elements, satisfying property P.1 of a good 6-coloring. To obtain a Hamiltonian path in the complement graph $G^{c}$, which complies with property P. 2 of a good 6-coloring, we will appropriately augment the corresponding Hamiltonian path of $G-v_{1}$ (which exists, by the induction hypothesis). To do so, it suffices to place $v_{1}$ anywhere between a pair of $c$-colored vertices along the Hamiltonian path of $G-v_{1}$. Hence, $G$ admits a good 6 -coloring.

To complete the proof in the inductive step, it remains to consider the case where each of $G-v_{1}, G-v_{2}, G-v_{3}$ and $G-v_{4}$ contain as subgraphs one of $K_{1,1, n_{0}-3}$, $K_{1, n_{0}-1}$ or $K_{2, n_{0}-2}$. By the pigeonhole principle, it follows that at least two out of them will contain the same subgraph. Assume w.l.o.g. that $G-v_{1}$ and $G-v_{2}$ contain the same subgraph. Since $\operatorname{deg}\left(v_{1}\right) \leq 5$ and $\operatorname{deg}\left(v_{2}\right) \leq 5$, it follows that $v_{1}$ and $v_{2}$ will belong to the big parts of $K_{1,1, n_{0}-3}, K_{1, n_{0}-1}$ or $K_{2, n_{0}-2}$. We consider the following subcases:

C.1: $G-v_{1}$ and $G-v_{2}$ contain $K_{1,1, n_{0}-3}$ as subgraphs: In this case, by planarity it follows that both copies of $K_{1,1, n_{0}-3}$ have the same poles, that is, their small parts consist of the same pair of vertices. This implies that $G$ contains $K_{1,1, n_{0}-2}$ as a subgraph, which is a contradiction.

C.2: $G-v_{1}$ and $G-v_{2}$ contain $K_{2, n_{0}-2}$ as subgraphs: In this case, planarity implies that both copies of $K_{2, n_{0}-2}$ have the same poles, which implies that $G$ contains $K_{2, n_{0}-1}$ as a subgraph, which is also a contradiction. 
C.3: $G-v_{1}$ and $G-v_{2}$ contain $K_{1, n_{0}-1}$ as subgraphs: Let $u_{i}$ be the vertex of $G-v_{i}$ that is adjacent to all the rest of its vertices, $i=1,2$. As already stated, $u_{i} \neq v_{i}$ holds, $i=1,2$. If $u_{1}=u_{2}$, then it follows that $G$ contains $K_{1, n_{0}}$ as subgraph, which is a contradiction. On the other hand, if $u_{1} \neq u_{2}$, then $\left\{u_{1}\right\},\left\{u_{2}\right\}$ and $V-\left\{u_{1}, u_{2}\right\}$ form a $K_{1,1, n_{0}-3}$ in $G$. By planarity, it follows that not both $G-v_{3}$ and $G-v_{4}$ can contain as subgraph one of $K_{1,1, n_{0}-3}, K_{1, n_{0}-1}$ or $K_{2, n_{0}-2}$; a contradiction.

From the above case analysis it follows that $G-v_{1}, G-v_{2}, G-v_{3}$ and $G-v_{4}$ cannot simultaneously contain as subgraphs one of $K_{1,1, n_{0}-3}, K_{1, n_{0}-1}$ or $K_{2, n_{0}-2}$ and the claim follows.

Consider now a planar graph $G$ with $n \geq 12$ vertices that admits a good 6-coloring. Next, we prove that $G$ is $(2, n)$-differential colorable. We color the vertices of $G$ with increasing numbers from 1 to $n$ according to the order in which they appear along the Hamiltonian path, say $P$, implied by the good 6 -coloring. We claim that this coloring corresponds to a valid $(2, n)$-differential coloring. Indeed, any two same-colored vertices that are consecutive along $P$ are not adjacent in $G$ (by the definition of 6 -coloring). In addition, when two edges of different colors are consecutive along $P$, then they are not connected by an edge in $G$, due to property P. 2 of a good 6 -coloring. Hence, the coloring implied by the good 6 -coloring of $G$ is indeed a valid $(2, n)$-differential coloring for $G$, as desired. We conlude with the following remark.

Remark. Using a simple ILP-formulation of the problem of finding a good 6-coloring, it can be shown that Theorem 3 holds for all planar graphs with $n \geq 12$ vertices.

It remains now to prove in detail the base of our induction. To this end, we first need to introduce some necessary notions. Let $W$ be a subset of the set of vertices of a graph $G=(V, E)$. A vertex $v$ is called simplicial to $W$, if every vertex in $W$ is a neighbour of $v$. When $W=V \backslash\{v\}$, we call $v$ a simplicial vertex of $G$. We denote the subgraph that $W$ induces in $G$ by $G[W]$. For $v, u \in V$ and $S$ a subgraph of $G$, by writing $v S u$ we mean a path in $G$ with vertex set $\{v, u\} \cup V(S)$ and endpoints $v, u$. We define $v S$ and $S u$ similarly.

Lemma 10. Let $T=\left(V_{T}, E_{T}\right)$ be a forest on $n$ vertices. Then, exactly one of the following is true:

i. $T$ is a tree with a vertex of degree $n-1$ (i.e., $T$ is a star), which implies that $T^{c}$ has no Hamiltonian path.

ii. $T$ is a tree with a vertex of degree $n-2$. In this case, $T^{c}$ contains a Hamiltonian path with $v$ as an endpoint. If in addition $\left|V_{T}\right| \geq 5$, then $T^{c}$ contains a path with $v$ as one endpoint and any other neighbour of $v$ in $T$ as second endpoint.

iii. $T$ has maximum degree at most $n-3$ and $T^{c}$ contains a Hamiltonian cycle.

Proof. The implication of (i1) is straight-forward. So, let us first prove that the implications of (ii) are also correct. To this end, assume that $T$ has a vertex $v$ of degree $n-2$. Since $T$ is tree and the degree of $v$ is $n-2$, it follows that $T$ has at least 4 vertices. 
More precisely, if $T$ has exactly 4 vertices, then $T$ is a path and therefore its complement is also a path, which starts or ends at $v$. If $\left|V_{T}\right| \geq 5$, let $u$ be the non-neighbour of $v$. Observe that $T^{c} \backslash\{v, u\}$ is a clique with at least three vertices. Let $w \in V_{T} \backslash\{v, u\}$. Since $\left|V_{T} \backslash\{v, u\}\right| \geq 3$, there is a $w^{\prime} \in V_{T} \backslash\{v, u\}$ with $w^{\prime} \neq w$, such that $w^{\prime}$ is a non-neighbour of $u$. Then $v u w^{\prime}\left(T^{c} \backslash\{v, u\}\right) w$ is a Hamiltonian path of $T^{c}$, which proves that the implications of (ii) is also correct.

To complete the proof it remains to prove that the complement of any forest $T$ with $n$ vertices, that contains no vertex of degree $n-1$ or $n-2$, has a Hamiltonian cycle. Our proof is by induction. The base of the induction corresponds to the case where $\left|V_{T}\right| \leq 4$. In this case, the maximum degree of $T$ is at most one and it is straightforward to see that $T^{c}$ is Hamiltonian.

For the inductive step, consider a forest $T$ with $\left|V_{T}\right| \geq 5$ and assume that the complements of all forests with $\left|V_{T}\right|-1$ vertices that do not satisfy (i) and (ii) are Hamiltonian. In this case, the radius of $T$ is at least 2. Let $r$ be a vertex of $T$ that achieves the radius of $T$. Since $T$ has no vertex of degree $n-2$, it follows that there are at least two leaves, say $u$ and $v$, that have distance to $r$ at least two. So, $T-v$ is not a star. If $T-v$ has a vertex of degree $n-3$, then $T \backslash\{u, v\}$ is a star with center $r$. Let $u^{\prime}$ and $v^{\prime}$ be the neighbours of $u$ and $v$ in $T$. Notice that $T \backslash\{r, u, v\}$ induces a clique in $T^{c}$. Hence, $r v u^{\prime}\left(T^{c} \backslash\{r, u, v\}\right) v^{\prime} u r$ is a Hamiltonian cycle in $T^{c}$. Consider now the case, where $T-v$ has no vertex of degree $n-3$. By the induction hypothesis, $T^{c}-v$ has a Hamiltonian cycle, say $C$. Since $|V(C)| \geq 4$ and the degree of $v$ in $T$ is one, there are two consecutive vertices $u$ and $u^{\prime}$ of $C$ that are non-adjacent to $v$ in $T$. Extending $C$ with $v$ by deleting edge $\left(u, u^{\prime}\right)$ and connecting $u$ and $u^{\prime}$ to $v$ implies a Hamiltonian cycle for $T^{c}$, which completes our inductive proof.

The contraction of an edge $e=(x, y)$ of $G$ is the identification of $x$ and $y$ into a new vertex $v_{e}$, adjacent to all former neighbours of $x$ and $y$. We say that $H$ is a minor of $G$ if $H$ can be obtained from $G$ by deleting edges and vertices, and by contracting edges. It is well-known that a graph $G$ is planar if and only if it does not contain $K_{5}$ or $K_{3,3}$ as a minor.

Lemma 11. Let $G=(V, E)$ be a planar graph and assume that the vertices of $G$ can be partitioned into two sets $C$ and $H$ such that: (i) $G[C]$ is biconnected outerplanar with at least 6 vertices and diameter at least 3 , and (ii) $G[H]$ has at least three vertices and $G[H]^{c}$ contains a Hamiltonian path. Then, $G^{c}$ contains a Hamiltonian path with at least one end-point in $C$.

Proof. By Lemma 6, there exists a Hamiltonian path $P_{c}$ of $G[C]^{c}$ with end-points $c$ and $c^{\prime}$, which are not consecutive on the outerface of $G[C]$. Let $P_{h}$ be a Hamiltonian path of $G[H]^{c}$ and denote by $h$ and $h^{\prime}$ its end-points. For a proof by contradiction assume that $G^{c}$ contains no Hamiltonian path with at least one end-point in $C$.

First, consider the case where $\left(h, h^{\prime}\right) \in E$. We claim that in graph $G$ vertices $h$ and $h^{\prime}$ cannot have more than two common neighbours from set $C$. To prove the claim, assume to the contrary that $w, w^{\prime}, w^{\prime \prime} \in C$ are three common neighbours of $h$ and $h^{\prime}$. Contract $C$ into a triangle with vertices $w, w^{\prime}, w^{\prime \prime}$. Then, $h, h^{\prime}, w, w^{\prime}$, and $w^{\prime \prime}$ form a $K_{5}$-minor in $G$, contradicting the planarity of $G$. Hence, $h$ and $h^{\prime}$ have at most two common neighbours from set $C$. Since $P_{c}$ has at least five edges, by pigeonhole 
principle it follows that there are two consecutive vertices, say $z$ and $z^{\prime}$ of $P_{c}$ (where $c, z z^{\prime}$ and $c^{\prime}$ appear in this order in $\left.P_{c}\right)$ such that $(h, z) \notin E$ and $\left(h^{\prime}, z^{\prime}\right) \notin E$. Hence, $c P_{c} z h P_{h} h^{\prime} z^{\prime} P_{c} c^{\prime}$ is a Hamiltonian path of $G^{c}$; a contradiction.

Next, consider the case where $\left(h, h^{\prime}\right) \notin E$. In this case, $P_{h}^{\prime}=P_{h} h h^{\prime}$ is a Hamiltonian cycle of $G[H]^{c}$. If $c$ and $c^{\prime}$ are not simplicial to $H$ in $G$, then we can just concatenate $P_{c}$ and $P_{h}^{\prime}$ through a non-edge in $G$ between an endpoint of $P_{c}$ and a vertex of $P_{h}^{\prime}$, and obtain a Hamiltonian path in $G^{c}$. Similarly, if $\left(c, c^{\prime}\right) \notin E(G)$, the $P_{c}^{\prime}=P_{c} c c^{\prime}$ is a Hamiltonian cycle of $G[C]^{c}$ and since $G$ excludes $K_{3,3}$ by planarity, we can always concatenate cycles $P_{c}^{\prime}$ and $P_{h}^{\prime}$ with a non-edge of $G$ between them and obtain a Hamiltonian path of $G^{c}$.

Therefore, we may assume that $\left(c, c^{\prime}\right) \in E$ and that for every $u \in H$, we have $(u, c) \in E$ and $\left(u, c^{\prime}\right) \in E$. Then, $\left(c, c^{\prime}\right)$ is a chord of $G[C]$. Let $A$ and $B$ be the two connected components of $G[C] \backslash\left\{c, c^{\prime}\right\}$. Notice that since the vertices of $A \cup B$ are the internal vertices of $P_{c}$, there exist $\alpha \in A$ and $\beta \in B$ such that $\alpha$ and $\beta$ are consecutive in $P_{c}$, where $c, \alpha, \beta, c^{\prime}$ appear with this order in $P_{c}$. Next, we make two claims. Our first claim is that both $\alpha$ and $\beta$ have at most two neighbours each from set $H$ in $G$. To prove the first claim assume to the contrary that one of $\alpha$ or $\beta$, say w.l.o.g $\alpha$, has three neighbours $w, w^{\prime}$ and $w^{\prime \prime}$ from set $H$ in $G$. Then, $\left\{\alpha, c, c^{\prime}\right\} \times\left\{w, w^{\prime}, w^{\prime \prime}\right\}$ is a $K_{3,3}$ in $G$, which contradicts the planarity of $G$. So, the first claim holds. Our second claim is that $\alpha$ and $\beta$ cannot have two common neighbours in $H$. Again, the proof is by contradiction. Let $w$ and $w^{\prime}$ be two such common neighbours of $\alpha$ and $\beta$. Then, vertices $\alpha, c, c^{\prime}, w$ and $w^{\prime}$ form a $K_{5}$-minor in $G$, contradicting the planarity of $G$. Since $P_{h}^{\prime}$ has at least three edges, it follows that there are two consecutive vertices $\gamma$ and $\delta$ of $P_{h}^{\prime}$ such that $(\alpha, \gamma) \notin E$ and $(\beta, \delta) \notin E$. This implies that $c P_{c} \alpha \gamma P_{h}^{\prime} \delta \beta P_{c} c^{\prime}$ is a Hamiltonian path of $G^{c}$, a contradiction. Since both cases, where $\left(h, h^{\prime}\right) \in E$ and $\left(h, h^{\prime}\right) \notin E$ were lead to a contradiction, the proof of the theorem follows.

The following lemma by Yang and Yuan [29] describes a structural property of planar graphs of maximum diameter two.

Lemma 12 (Yang and Yuan [29]). Let $G$ be a planar graph of diameter at most two 2. Then, the vertices of $G$ can be partitioned into two sets such that each one induces a forest in $G$.

Finally, the following lemma will allow us to obtain a good 6-coloring of a planar graph with $n=12$ vertices, which does not contain as subgraphs $K_{1,11}, K_{2,10}$ and $K_{1,1,9}$.

Lemma 13. Let $G=(V, E)$ be planar on $n=12$ vertices, which does not contain as subgraphs $K_{1,11}, K_{2,10}$ and $K_{1,1,9}$. Then, $G^{c}$ contains a Hamiltonian path.

Proof. It is enough to prove the lemma for maximal planar graphs with $n=12$ vertices, since any planar graph, which does not contain as subgraphs $K_{1,11}, K_{2,10}$ and $K_{1,1,9}$, can be augmented to maximal by adding edges and simultaneously avoiding creating $K_{1,11}, K_{2,10}$ and $K_{1,1,9}$. So, we will assume w.l.o.g. that $G$ is maximal planar with a fixed planar embedding. Moreover, to keep notational consistency with the rest of the section so far, from now on we will prefer to refer to the obstructions in the 
statement of the Lemma as $K_{1, n-1}, K_{2, n-2}$ and $K_{1,1, n-3}$ instead of $K_{1,11}, K_{2,10}$ and $K_{1,1,9}$. We distinguish two main cases based on the diameter of $G$ :

C.1: The diameter of $G$ is at least 3: Let $u$ and $v$ be two vertices of $G$ such that $d(u, v)=\operatorname{diam}(G)$. Since $G$ is maximal planar, it is 3-connected. Hence, there are at least three internally vertex-disjoint paths from $u$ to $v$. Let $P_{1}, P_{2}$ and $P_{3}$ be three such paths chosen such that $\left|V\left(P_{1}\right)\right|+\left|V\left(P_{2}\right)\right|+\left|V\left(P_{3}\right)\right|$ is minimum, where $V\left(P_{i}\right)$ denotes the set of vertices of path $P_{i}, i=1,2,3$. Since $d(u, v) \geq 3$, it follows that each of $P_{1}, P_{2}$ and $P_{3}$ consists of at least 4 vertices and by their choice, they must be induced in $G$.

Let $i, j \in\{1,2,3\}$ be such that $i \neq j$. Denote by $C_{i j}$, to be called the interior of $P_{i} \cup P_{j}$, the vertices of $G$ contained in the open region of $G \backslash\left(P_{i} \cup P_{j}\right)$ that does not contain $P_{6-i-j}$. Similarly, denote by $D_{i j}$, which we call the exterior of $P_{i} \cup P_{j}$, the set of all vertices of $G$ contained neither in the interior of $P_{i} \cup P_{j}$ nor in $P_{i} \cup P_{j}$. In other words, $D_{i j}:=V \backslash\left(V\left(P_{i}\right) \cup V\left(P_{j}\right) \cup C_{i j}\right)$. By definition and the planarity of $G, P_{i} \cup P_{j}$ separates $C_{i j}$ from $D_{i j}$. Since $D_{i j}$ contains the internal vertices of $P_{6-i-j}$, it follows that $\left|D_{i j}\right| \geq 2$. Additionally, $C_{12} \cup C_{23} \cup C_{31}=$ $V \backslash\left(V\left(P_{1}\right) \cup V\left(P_{2}\right) \cup V\left(P_{3}\right)\right)$. We also denote by $G_{i j}$ the graph induced by $P_{i} \cup P_{j}$, that is, $G_{i j}=G\left[P_{i} \cup P_{j}\right]$. Recall that the paths $P_{1}, P_{2}, P_{3}$ are induced and that $P_{6-i-j}$ separates the region that contains the vertices of $D i j$, hence all the edges of $G_{i j}$ not on $P_{i}$ or $P_{j}$ must be embedded in the region containing the vertices of $C_{i j}$. It follows that $G_{i j}$ is outerplanar. In addition, $G_{i j}$ does not contain $K_{1, n-1}$ as a subgraph (because $G_{i j}$ is a subgraph of $G$ ) and is of diameter at least three (because of the choice of $u$ and $v$ ). By Lemma 6 it follows that $G_{i j}^{c}$ contains a Hamiltonian path (observe that the graph of Figure 3e has diameter two, so it cannot be isomorphic to $G_{i j}$ ).

We define the $i j$-discrepancy to be the quantity $\delta_{i j}:=|| C_{i j}|-| D_{i j}||$. The existence of an $i j$-discrepancy at most 1 immediately allows us to find a Hamiltonian path in $G^{c}$ as follows: alternate between the vertices of $C_{i j}$ and $D_{i j}$ to obtain a Hamiltonian path of $G^{c}\left[C_{i j} \cup D_{i j}\right]$ and apply Lemma 11 to $G_{i j}$ and $G\left[C_{i j} \cup D_{i j}\right]$. Whenever one of the three discrepancies is at most 1 , we will call a path constructed as above a standard path in $G^{c}$. It remains to consider the cases where all three $i j$-discrepancies are at least 2 . We distinguish three main subcases based on the maximum length of the paths $P_{1}, P_{2}$ and $P_{3}$.

C.1.1: $\max \left\{\left|V\left(P_{i}\right)\right|: i=1,2,3\right\}=4$ : Since $d(u, v) \geq 3$, it follows that $\left|V\left(P_{1}\right)\right|=\left|V\left(P_{2}\right)\right|=\left|V\left(P_{3}\right)\right|=4$. Since all three exteriors contain at least two vertices, the only possible way that all $i j$-discrepancies are at least 2 is that an interior, say $C_{12}$, contains all 4 remaining vertices $v_{1}, v_{2}, v_{3}, v_{4}$ of $G$ not on a $P_{i}$ path. If the four vertices induce a $K_{4}$, then by the planarity of $G$, one of them, say $v_{1}$, will be the only vertex in one of the two open regions in the plane defined by removing the triangle of the remaining three vertices $v_{2}, v_{3}, v_{4}$. Therefore, $v_{1}$ is only adjacent to $v_{2}, v_{3}, v_{4}$. In this case, since the discrepancy between $C_{12} \backslash\left\{v_{1}\right\}$ and $D_{12}$ is 1 , we can consider a standard path in $G^{c} \backslash\left\{v_{1}\right\}$ with one end-point in $V\left(G_{12}\right)$ and complete it to a Hamiltonian path for $G^{c}$ with $v_{1}$. 
Otherwise, there are two of the four vertices, say $v_{1}, v_{2}$, that are nonadjacent in $G$. Let $D_{12}=\left\{u_{1}, u_{2}\right\}$. Then $G^{c}\left[C_{i j} \cup D_{i j}\right]$ has the Hamiltonian path $v_{3} u_{1} v_{1} v_{2} u_{2} v_{4}$ and the Hamiltonian path of $G^{c}$ follows by Lemma 11 .

C.1.2: $\max \left\{\left|V\left(P_{i}\right)\right|: i=1,2,3\right\}=5$ : In this case, there are only three cases for all three $i j$-discrepancies to be at least 2 :

(i) $\left|V\left(P_{1}\right)\right|=5,\left|V\left(P_{2}\right)\right|=\left|V\left(P_{3}\right)\right|=4,\left|C_{12}\right|=\left|C_{23}\right|=\left|C_{31}\right|=1$,

(ii) $\left|V\left(P_{1}\right)\right|=4,\left|V\left(P_{2}\right)\right|=\left|V\left(P_{3}\right)\right|=5,\left|C_{12}\right|=\left|C_{31}\right|=1$, and

(iii) $\left|V\left(P_{1}\right)\right|=\left|V\left(P_{2}\right)\right|=\left|V\left(P_{3}\right)\right|=5,\left|C_{12}\right|=1,\left|C_{23}\right|=\left|C_{31}\right|=0$.

We study each of these three cases separately in the following.

(i) Since each exterior consists of a single vertex, we may assume that $C_{i j}=\left\{v_{i j}\right\}$. Note that $v_{12}, v_{23}, v_{31}$ form an independent set in $G$. Now, let $u^{\prime}$ and $v^{\prime}$ be the unique neighbours of $u$ and $v$ in $P_{1}$, respectively. Let also $w$ be the middle vertex of $P_{1}$. Recall that $P_{1}$ is induced in $G$. Hence, $u^{\prime}$ and $v^{\prime}$ are not adjacent. If $v_{12}, u^{\prime}, v_{31}$ and $v^{\prime}$ induce a cycle $C$ in $G$, then $w$ is the unique vertex contained in one of the two open regions of the plane after removing $C$. Hence, $w$ is adjacent only to the vertices of $C$. Notice that vertices $u^{\prime}, v^{\prime}$, $v_{23}, v_{12}$ and $v_{31}$ form a path in $G^{c}$. By Lemma 11 for $G_{23}$ and the rest of the vertices of $G-w$, we obtain a Hamiltonian path $P$ for $G-w$ with one endpoint in $V\left(G_{23}\right)$. We complete $P$ by connecting the end-point of $P$ in $V\left(G_{23}\right)$ to $w$ to obtain a Hamiltonian path of $G^{c}$. On the other hand, if $v_{12}, u^{\prime}, v_{31}$ and $v^{\prime}$ do not induce a cycle $C$ in $G$, then there is an edge of $C$ missing. Assume w.l.o.g. that $\left(v_{23}, u^{\prime}\right) \notin E$. In this case, vertices $v_{31}, v_{12}, u^{\prime}, v^{\prime}, v_{23}$ and $w$ form a path in $G^{c}$ and we apply Lemma 11 to obtain a Hamiltonian path for $G^{c}$.

(ii) As in the previous subcase, let $C_{12}=\left\{v_{12}\right\}$ and $C_{31}=\left\{v_{23}\right\}$. Let $u^{\prime}, w$ and $v^{\prime}$ be the internal vertices of $P_{3}$, where $u^{\prime}$ and $v^{\prime}$ are the neighbours of $u$ and $v$ in $P_{3}$, respectively. Recall that $P_{3}$ is induced. Hence, $\left(u^{\prime}, v^{\prime}\right) \notin E$. It follows that vertices $w, v_{12}, u^{\prime}$ and $v^{\prime}$ form a Hamiltonian path of $G^{c}\left[C_{12} \cup D_{12}\right]$. To obtain a Hamiltonian path for $G^{c}$, we apply Lemma 11 to $G_{12}$ and $G\left[C_{12} \cup D_{12}\right]$.

(iii) Exactly as in the previous subcase, vertices $w, v_{12}, u^{\prime}$ and $v^{\prime}$ form a Hamiltonian path of $G^{c}\left[C_{12} \cup D_{12}\right]$. So, in order to obtain a Hamiltonian path for $G^{c}$, we apply Lemma 11 to $G_{12}$ and $G\left[C_{12} \cup D_{12}\right]$.

C.1.3: $\max \left\{\left|V\left(P_{i}\right)\right|: i=1,2,3\right\} \geq 6$ : Suppose w.l.o.g. that $\left|V\left(P_{1}\right)\right| \geq 6$. Let $P_{1}^{\prime}$ be the path consisting of the internal vertices of $P_{1}$. Since $P_{1}$ is induced, $P_{1}^{\prime c}$ contains a Hamiltonian path by Lemma 10 . Now, observe that $\left|C_{12} \cup C_{23} \cup C_{31}\right|=\left|V \backslash V\left(P_{1} \cup P_{2} \cup P_{3}\right)\right| \leq 2$. We now claim that every vertex in $C_{12} \cup C_{23} \cup C_{31}$ has at most three neighbours in $V\left(P_{1}^{\prime}\right)$. To prove this claim, assume to the contrary that $w \in C_{12} \cup C_{31}$ has at least four neighbours in $V\left(P_{1}^{\prime}\right)$. If this is the case, we can replace $P_{1}$ with a shorter path between $u$ and $v$ through $w$, which contradicts the choice of 
$P_{1}, P_{2}$ and $P_{3}$. Hence, every vertex in $C_{12} \cup C_{23} \cup C_{31}$ has at most three neighbours in $V\left(P_{1}^{\prime}\right)$. We study each of these three cases implied by our earlier observation that $\left|C_{12} \cup C_{23} \cup C_{31}\right| \leq 2$ in the following.

(i) $\left|C_{12} \cup C_{23} \cup C_{31}\right|=0$ : In this case, $G^{c}\left[C_{23} \cup D_{23}\right]$ is the graph $P_{1}^{\prime c}$. Hence, $G^{c}\left[C_{23} \cup D_{23}\right]$ contains a Hamiltonian path. To obtain a Hamiltonian path for $G^{c}$, we apply Lemma 11 to $G_{23}$ and $G\left[C_{23} \cup\right.$ $\left.D_{23}\right]$.

(ii) $\left|C_{12} \cup C_{23} \cup C_{31}\right|=1$ : Let $x$ be the unique vertex of $C_{12} \cup C_{23} \cup C_{31}$. We consider the two cases where $P_{1}^{\prime}$ has either at least 5 vertices or exactly 4 vertices.

In the former case, $P_{1}^{\prime c}$ contains a Hamiltonian cycle and we connect $x$ to a vertex of $P_{1}^{\prime c}$ in $G^{c}$. Since $G^{c}\left[C_{23} \cup D_{23}\right]$ is the graph $G^{c}\left[V\left(P_{1}^{\prime}\right) \cup\{x\}\right]$, we apply Lemma 11 to $G_{23}$ and $G\left[C_{23} \cup D_{23}\right]$ to obtain a Hamiltonian path for $G^{c}$.

Consider now the latter case, in which $P_{1}^{\prime}$ has exactly 4 vertices. In this case, $P_{2}$ has exactly five vertices, while $P_{3}$ has four vertices. We distinguish three subcases based on whether $x$ belongs to $C_{12}$, to $C_{23}$ or to $C_{31}$. Assume first that $x$ belongs to $C_{23}$, that is, $C_{23}=\{x\}$. It follows that $G^{c}\left[C_{23} \cup D_{23}\right]$ is the graph $G^{c}\left[V\left(P_{1}^{\prime}\right) \cup\{x\}\right]$ and clearly $G^{c}\left[C_{23} \cup D_{23}\right]$ contains a Hamiltonian path. A Hamiltonian path for $G^{c}$ follows by Lemma 11 for $G_{23}$ and $G\left[C_{23} \cup D_{23}\right]$. So, next assume that $x$ belongs to $C_{12}$, that is, $C_{12}=\{x\}$. In this case, the 12-discrepancy is 1 and as stated earlier $G^{c}$ has a Hamiltonian path. Finally, we consider the case where $x$ belongs to $C_{31}$, that is, $C_{31}=\{x\}$. Let $P_{2}^{\prime}$ be the path consisting of the internal vertices, say $u^{\prime}, w$ and $v^{\prime}$, of $P_{2}$, where $u^{\prime}$ and $v^{\prime}$ are the neighbor of $u$ and $v$, respectively. Then, vertices $w, x u^{\prime}$ and $v^{\prime}$ form a Hamiltonian path of $G^{c}\left[C_{31} \cup D_{31}\right]$. To obtain a Hamiltonian path for $G^{c}$, we apply Lemma 11 to $G_{31}$ and $G\left[C_{31} \cup D_{31}\right]$.

(iii) $\left|C_{12} \cup C_{23} \cup C_{31}\right|=2$ : Assume that $C_{12} \cup C_{23} \cup C_{31}=\left\{w, w^{\prime}\right\}$. The only possible way to have that all three discrepancies are at least 2, is when $C_{23}=\left\{w, w^{\prime}\right\}$. Hence there are no edges in $G$ between $\left\{w, w^{\prime}\right\}$ and $V\left(P_{1}\right)$. Since $P_{1}^{\prime c}$ has a Hamiltonian path, $G^{c}\left[C_{23} \cup D_{23}\right]$, which is the graph $G^{c}\left[V\left(P_{1}^{\prime}\right) \cup\left\{w, w^{\prime}\right\}\right]$, contains a Hamiltonian path. Hence, to obtain a Hamiltonian path for $G^{c}$, we apply Lemma 11 to $G_{23}$ and $G\left[C_{23} \cup D_{23}\right]$.

C.2: The diameter of $G$ is 2: By Lemma 12, there is a partition of $V$ into two sets $V_{1}$ and $V_{2}$, such that both $T_{1}=G\left[V_{1}\right]$ and $T_{2}=G\left[V_{2}\right]$ are forests. Let $n_{1}=$ $\left|V\left(T_{1}\right)\right|$ and $n_{2}=\left|V\left(T_{2}\right)\right|$. The degenerate cases where $\min \left\{n_{1}, n_{2}\right\} \leq 2$ are straightforward and left as an exercise to the reader. So, we may assume in what follows that $\min \left\{n_{1}, n_{2}\right\} \geq 3$. We consider the following subcases:

C.2.1: $T_{1}$ and $T_{2}$ are stars: Let $r_{1}$ and $r_{2}$ be the centers of $T_{1}$ and $T_{2}$, respectively. Then, $T_{1}^{c}-r_{1}$ and $T_{2}^{c}-r_{2}$ are complete subgraphs of $G^{c}$. Hence, they have Hamiltonian cycles where the vertices appear in any possible order. 
A linking path of $G^{c}$ is a path of the form $t_{1} r_{2} r_{1} t_{2}$ in $G^{c}$, where $t_{1}$ and $t_{2}$ are two vertices of $T_{1}$ and $T_{2}$, respectively. If a linking path exists, then $\left(T_{1}^{c}-r_{1}\right) t_{1} r_{2} r_{1} t_{2}\left(T_{2}^{c}-r_{2}\right)$ gives us a Hamiltonian path in $G^{c}$. So, we may assume that such a path does not exist in $G^{c}$. On the other hand, since $G$ does not contain simplicial vertices, none of $r_{1}$ and $r_{2}$ are simplicial to $V\left(T_{2}\right)$ and $V\left(T_{1}\right)$, respectively. So, there are two vertices $t_{1}$ and $t_{2}$ of $T_{1}$ and $T_{2}$, respectively, such that $\left(r_{1}, t_{2}\right) \notin E$ and $\left(r_{2}, t_{1}\right) \notin E$. Since $G$ does not contain $K_{2, n-2}$ as a subgraph, it follows that $\left\{t_{1}, t_{2}\right\} \neq\left\{r_{1}, r_{2}\right\}$. We assume w.l.o.g. that $t_{1} \neq r_{1}$. A linking edge of $G^{c}$ is an edge $\left(s_{1}, s_{2}\right)$ of $G^{c}$, where $s_{1}$ is a vertex of $T_{1}$ different from $r_{1}$ and $t_{1}$ and $s_{2}$ is a vertex of $T_{2}$ different from $r_{2}$ and $t_{2}$. We distinguish two cases based on whether $t_{2}$ is the vertex $r_{2}$ or not.

(i) $t_{2} \neq r_{2}$ : In this case, $\left(r_{1}, r_{2}\right)$ is an edge of $G$, as otherwise $t_{1} r_{2} r_{1} t_{2}$ is a linking path in $G^{c}$. Suppose that there are no linking edges in $G^{c}$, that is, if $\tau_{1}$ and $\tau_{2}$ are two vertices of $T_{1}$ and $T_{2}$ not in $\left\{r_{1}, t_{1}\right\}$ and $\left\{r_{2}, t_{2}\right\}$, respectively, then $\left(\tau_{1}, \tau_{2}\right)$ is an edge of $G$. If $\min \left\{n_{1}, n_{2}\right\}=3$ (w.l.o.g say $T_{1}=\left\{r_{1}, t_{1}, s_{1}\right\}$ ), then $G$ contains a $K_{1,1, n-3}$ with parts $\left\{s_{1}\right\},\left\{r_{2}\right\}$ and $V \backslash\left\{s_{1}, r_{2}, t_{2}\right\}$, a contradiction. If $\min \left\{n_{1}, n_{2}\right\} \geq 4$, then $G$ clearly contains a $K_{3,3}$, a contradiction to its planarity. Therefore, there exists a linking edge $\left(s_{1}, s_{2}\right)$ in $G^{c}$. Then $G^{c}$ contains a Hamiltonian path of the form $r_{2} t_{1}\left(T_{1}^{c}-r_{1}\right) s_{1} s_{2}\left(T_{2}^{c}-r_{2}\right) t_{2} r_{1}$.

(ii) $t_{2}=r_{2}$ : In this case, we may assume that $r_{1}$ is adjacent to all leaves of $T_{2}$, as otherwise we can transform this case into the previous one. Again, the presence of a linking edge $\left(s_{1}, s_{2}\right)$ in $G^{c}$ allows for a Hamiltonian path in $G^{c}$ that is of the form $r_{1} r_{2} t_{1}\left(T_{1}^{c}-r_{1}\right) s_{1} s_{2}\left(T_{2}^{c}-\right.$ $\left.r_{2}\right)$. Hence, we may assume that no linking edge exists. By symmetry, $r_{2}$ must also be adjacent to all vertices of $T_{1}$ not in $\left\{r_{1}, t_{1}\right\}$. Then, if $n_{2} \geq 4$, then $G$ contains $K_{3,3}$ (with parts $\left\{r_{1}, t_{1}, s_{1}\right\}$ and $r_{2}$ together with two any leaves of $T_{2}$ ) as a subgraph, a contradiction to its planarity. Otherwise, $n_{2}=3$ implies that $T_{2}$ consists of $r_{2}, t_{2}$ and $s_{2}$. In this case, $G$ contains again $K_{3,3}$ (with parts $\left\{r_{2}, t_{2}, s_{2}\right\}$ and any three leaves of $T_{1}$ ) as a subgraph, again a contradiction. So, $G$ must have at least one linking edge and therefore $G^{c}$ contains a Hamiltonian path.

C.2.2: Neither $T_{1}$ nor $T_{2}$ is a star: In this case, each of $T_{1}$ and $T_{2}$ has to satisfy either condition (iii) or condition (iii) of Lemma 10 We consider the corresponding cases in the following.

(i) Both $T_{1}$ and $T_{2}$ satisfy condition (iiii) of Lemma 10 Let $u_{1} \ldots u_{n_{1}} u_{1}$ and $v_{1} \ldots v_{n_{2}} v_{1}$ be the Hamiltonian cycles implied by Lemma 10 of $T_{1}^{c}$ and $T_{2}^{c}$, respectively. Since $n_{1}, n_{2} \geq 3$, it follows by the planarity of $G$ that not all edges between the vertices of $T_{1}$ and the vertices of $T_{2}$ are present in $G$. Assume w.l.o.g. that $\left(u_{n_{1}}, v_{1}\right) \notin E$. In this case, $u_{1} \ldots u_{n_{1}} v_{1} \ldots v_{n_{2}}$ is a Hamiltonian path of $G^{c}$. 
(ii) $T_{1}$ satisfies condition (ii) and $T_{2}$ satisfies condition (iii): As in the previous case, let $C=v_{1} \ldots v_{n_{2}} v_{1}$ be the Hamiltonian cycle of $T_{2}^{c}$ implied by Lemma 10 . Since $T_{1}$ is not a star, it follows that $n_{1} \geq 4$. Let $r_{1}$ be the vertex of $T_{1}$ with degree $n_{1}-2$ and $s_{1}$ the vertex of $T_{1}$, which is not neighboring with $r_{1}$. Then, $T_{1}^{c}$ has a Hamiltonian path $P$ which starts from vertex $r_{1}$, then visits vertex $s_{1}$, then visits any leaf of $T_{1}$ other than $s_{1}$, then the common neighbor of $r_{1}$ and $s_{1}$ and then the remaining leaves of $T_{1}$ in any order (if any). Denote by $u_{1}$ the end-point $P$, that is different from $r_{1}$. We can assume that all edges between $\left\{r_{1}, u_{1}\right\}$ and the vertices of $T_{2}$ exist in $G$, as otherwise we could appropriately concatenate $P$ and $C$ to obtain a Hamiltonian path of $G^{c}$. Since $G$ does not contain $K_{3,3}$ as a subgraph, all vertices of $T_{1}$ other than $r_{1}$ and $u_{1}$ must have at most two neighbours in $T_{2}$. Observe that if there are two consecutive internal vertices $x_{1}$ and $x_{2}$ of $P$ and two consecutive vertices $y_{1}$ and $y_{2}$ of $C$ such that $\left(x_{1}, y_{1}\right) \notin$ $E$ and $\left(x_{2}, y_{2}\right) \notin E$, then $r_{1} P x_{1} y_{1} C y_{2} x_{2} P u_{1}$ is a Hamiltonian path of $G^{c}$. If $n_{2} \geq 4$, this is always true by the pigeonhole principle for any two internal vertices of $P$. Consider now the case where $n_{2}=3$ and assume that the vertices of $T_{2}$ are $t_{1}, t_{2}$ and $t_{3}$. In this case, the approach mentioned above is not applicable only when every internal vertex of $P$ is adjacent to exactly the same two vertices of $T_{2}$, say w.1.o.g. to $t_{1}$ and $t_{2}$. In this particular case, however, $G$ contains $K_{3,3}$ (with parts $\left\{r_{1}, t_{1}, t_{2}\right\}$ and tree internal vertices of $P$ ) as a subgraph, a contradiction to its planarity. Note that the case where $T_{2}$ satisfies condition (ii) and $T_{1}$ satisfies condition (iii) is symmetric.

(iii) Both $T_{1}$ and $T_{2}$ satisfy condition (ii) of Lemma 10 . Since $T_{1}$ and $T_{2}$ are not stars, it follows that $n_{1}, n_{2} \geq 4$. Let $r_{1}$ and $r_{2}$ be the vertices of $T_{1}$ and $T_{2}$ with degrees $n_{1}-2$ and $n_{2}-2$, respectively. Let also $s_{1}$ and $s_{2}$ be the vertices of $T_{1}$ and $T_{2}$ that are not neighbors of $r_{1}$ and $r_{2}$ in $T_{1}$ and $T_{2}$, respectively. If both $n_{1}$ and $n_{2}$ are at least 5 , then $T_{1}$ and $T_{2}$ contain at least three vertices each that are all different from $r_{1}, s_{1}, r_{2}, s_{2}$. Since $G$ does not contain $K_{3,3}$ as a subgraph, it follows that there exist two vertices $w_{1}$ and $w_{2}$ in $T_{1}$ and $T_{2}$, respectively, such that $w_{1} \notin\left\{r_{1}, s_{1}\right\}, w_{2} \notin\left\{r_{2}, s_{2}\right\}$ and $\left(w_{1}, w_{2}\right) \notin E$. By Lemma 10 (ii), there are Hamiltonian paths of $T_{1}^{c}$ and $T_{2}^{c}$ with end-points $r_{1}$ and $w_{1}$ on one hand, and $r_{2}$ and $w_{2}$ on the second hand. Concatenating them through $w_{1}$ and $w_{2}$, we obtain a Hamiltonian path of $G^{c}$.

If $n_{1}$ or $n_{2}$ is less than 5 , then either $T_{1}$ or $T_{2}$, say w.l.o.g. $T_{1}$, is a path on four vertices (recall that $n_{1}, n_{2} \geq 4$ ). Let $v_{1}, v_{2}, v_{3}$ and $v_{4}$ be the vertices of $T_{1}$ as they appear along the path and define $r_{2}$ and $s_{2}$ for $T_{2}$ as above. Notice that $T_{1}^{c}$ is uniquely defined as the path connecting vertices $v_{2}, v_{4}, v_{1}$ and $v_{3}$ in this order. We now claim that $v_{2}$ and $v_{3}$ cannot be both simplicial to the vertices of $T_{2}$ other than $r_{2}$ and $s_{2}$. Assume that this is not the case. Then, $G$ would contain 
$K_{3,3}$ (with parts $\left\{v_{2}, v_{3}, r_{2}\right\}$ and three vertices of $T_{2}$ other than $r_{2}$ and $s_{2}$ ) as a subgraph, a contradiction to its planarity. So, the claim holds and we can assume w.l.o.g. that there exists a vertex $w$ of $T_{2}$ other than $r_{2}$ and $s_{2}$ such that $\left(w, v_{1}\right) \notin E$. Then we concatenate $T_{1}^{c}$ with a Hamiltonian path of $T_{2}^{c}$ through $v_{1}$ and $w$ and obtain a Hamiltonian path of $G^{c}$.

C.2.3: $T_{1}$ is a star and $T_{2}$ is not a star: Let $r_{1}$ be the center of $T_{1}$ and observe again that $T_{1}^{c}-r_{1}$ is a complete graph. Since $G$ does not contain $K_{1, n-1}$ as a subgraph, there is a vertex $t_{2}$ of $T_{2}$ that is not adjacent to $r_{1}$ in $G$, that is, $\left(r_{1}, t_{2}\right) \notin E$. Moreover, if $T_{2}$ has three vertices it is always a subgraph of a star, hence this case would be an easier version of C.2.1. Therefore, we may assume that $T_{2}$ has at least four vertices. Note that $T_{2}$ satisfies either condition (iii) or condition (iii) of Lemma 10 . We investigate each of these two cases in what follows.

(i) $T_{2}$ satisfies condition (iii) of Lemma 10 Let $r_{2}$ be the vertex of $T_{2}$ with degree $n_{2}-2$ and denote by $\left\{\tau_{1}, \ldots, \tau_{n_{2}-2}\right\}$ the neighbourhood of $r_{2}$ in $T_{2}$. Let also $s_{2}$ be the vertex of $T_{2}$ that is not neighboring $r_{2}$ in $T_{2}$. Hence, $s_{2}$ is either a leaf or an isolated vertex of $T_{2}$. So, we can assume w.l.o.g. that $s_{2}$ is not adjacent to $\left\{\tau_{1}, \ldots, \tau_{n_{2}-3}\right\}$. Moreover, notice that the neighbourhood of $r_{2}$ in $T_{2}$ consists of at least two vertices and that it induces a clique in $T^{c}$. We now claim that not all edges between $\left\{\tau_{1}, \ldots, \tau_{n_{2}-2}\right\}$ and the leaves of $T_{1}$ are present in $G$. For a proof by contradiction, assume that this is not the case. We consider two subcases. If $T_{1}$ has more than two leaves, then $G$ would contain $K_{3,3}$ as a subgraph (indeed, any subset of $\left\{r_{1}, \tau_{1}, \ldots, \tau_{n_{2}-2}\right\}$ with three elements together with any three leaves of $T_{1}$ would yield a $K_{3,3}$ in $G$ ), a contradiction to its planarity. If $T_{1}$ has exactly two leaves, then $n_{2}=9$ and $G$ contains a $K_{3,3}$ with parts the two leaves of $T_{1}$ together with $r_{2}$ and any three vertices of $\left\{\tau_{1}, \ldots, \tau_{n_{2}-2}\right\}$. So, our claim holds. Hence, there exist a leaf $w$ of $T_{1}$ and a vertex $z \in\left\{\tau_{1}, \ldots, \tau_{n_{2}-2}\right\}$ that are not adjacent in $G$, that is, $(w, z) \notin E$.

Observe that $r_{2}$ cannot be adjacent to all the vertices of $T_{1}$, because $G$ would contain a $K_{1,1, n-3}$. If $t_{2}=r_{2}$, then there is a Hamiltonian path in $G^{c}$ that is of the form $r_{1} r_{2} T_{2}^{c} z w\left(T_{1}^{c}-r_{1}\right)$, as desired. Otherwise, $r_{2}$ is adjacent to $r_{1}$ in $G$. In this case, by planarity it follows that there exists a leaf $w^{\prime}$ of $T_{1}$ that is different from $w$, such that $r_{2}$ is not adjacent to $w^{\prime}$, that is $\left(r_{2}, w^{\prime}\right) \notin E$. If $t_{2} \neq s_{2}$, then $G^{c}$ contains a Hamiltonian path that is of the form $r_{1} t_{2} T_{2}^{c} r_{1} w\left(T_{1}^{c}-r_{1}\right)$. Otherwise if $t_{2}=s_{2}, G^{c}$ contains a Hamiltonian path that is of the form $r_{2} w^{\prime}\left(T_{1}^{c}-r_{1}\right) w z\left(T_{2}^{c}-r_{2}\right) t_{2} r_{1}$. From the above, it follows that if $T_{2}$ satisfies condition (iii) of Lemma 10 , then there is always a Hamiltonian path in $G^{c}$.

(ii) $T_{2}$ satisfies condition (iii) of Lemma 10 Let $C=v_{1} \ldots v_{n_{2}} v_{1}$ be a Hamiltonian cycle of $T_{2}^{c}$. We will assume w.l.o.g. that $v_{1}=t_{2}$. Sup- 
pose first that $n_{1} \geq 4$. Then, if both $v_{2}$ and $v_{n_{2}}$ are simplicial to all vertices of $T_{1}$ other than $r_{1}$, then $G$ has $K_{3,3}$ (with parts $\left\{r_{1}, v_{2}, v_{n_{2}}\right\}$ and three vertices of $T_{1}$ other than $r_{1}$ ) as subgraph, a contradiction to its planarity. Therefore, there is a vertex $w$ of $T_{1}$ other than $r_{1}$ such that w.l.o.g. $\left(w, v_{n_{2}}\right) \notin E$. We find a Hamiltonian cycle in $G^{c}$ by starting with $r_{1} v_{1} v_{2} \ldots v_{n_{2}} w$ and traversing the rest of $T_{1}^{c}-r_{1}$.

Otherwise, $n_{1}=3$ and we can assume that $T_{1}$ consists of vertices $r_{1}$, $t_{1}$ and $s_{1}$. Notice that if both $t_{1}$ and $s_{1}$ are simplicial to the vertices of $T_{2}$, then $G$ contains $K_{2, n-2}$ as a subgraph (with poles $t_{1}$ and $s_{1}$ ). Hence, there is a vertex $v_{i}$ of $C$, such that w.l.o.g. $\left(s_{1}, v_{i}\right) \notin E$. Now, observe that if there is a vertex $x$ of $T_{2}$ that has at most one neighbour in $T_{1}$, then $T_{1} \cup\{x\}$ and $T_{2}-x$ are two forests that define a partition of $V$, which belongs to a case that we have already considered. So, we may assume that every vertex of $T_{2}$ has at least two neighbours in $T_{1}$.

It follows that for $v_{1}=t_{2}$, we have that $\left(t_{1}, v_{1}\right) \in E$ and $\left(s_{1}, v_{1}\right) \in$ $E$. Moreover, if there are two consecutive vertices $v_{j}$ and $v_{j+1}$ of $C$ that are not adjacent to $r_{1}$, then $t_{1} s_{1} v_{i} C v_{j} r_{1} v_{j+1} C v_{i-1}$ is a Hamiltonian path of $G^{c}$. Since $\left(r_{1}, v_{1}\right) \notin E$, we may assume that $\left(r_{1}, v_{2}\right) \in E$ and $\left(r_{1}, v_{n_{2}}\right) \in E$. In the following, we may assume that all edges between $\left\{t_{1}, s_{1}\right\}$ and $\left\{v_{2}, v_{n_{2}}\right\}$ must be present in $G$. To see this assume that $\left(s_{1}, v_{2}\right) \notin E$. The remaining cases are symmetric. Then $t_{1} s_{1} v_{2} C v_{1} r_{1}$ is a hamiltonial path of $G^{c}$. Now, suppose that $\left(v_{2}, v_{n_{2}}\right) \in E$. Then by contracting $v_{1}$ on $s_{2}$ we obtain a $K_{5}$-minor in $G$ with vertices $r_{1}, s_{1}, t_{1}, v_{2}, v_{n_{2}}$, a contradiction. It follows that $\left(v_{2}, v_{n_{2}}\right) \notin E$. If $\left(v_{3}, s_{1}\right) \notin E$, then $t_{1} s_{1} v_{3} C v_{n_{2}} v_{2} v_{1} r_{1}$ is a hamiltonian path of $G^{c}$. Symmetrically, if $\left(v_{3}, t_{1}\right) \notin E$, then $s_{1} t_{1} v_{3} C v_{n_{2}} v_{2} v_{1} r_{1}$ is a hamiltonian path of $G^{c}$. So, we may further assume that $\left(v_{3}, t_{1}\right) \in E$ and $\left(v_{3}, s_{1}\right) \in E$. So, since the graph on vertices $r_{1}, t_{1}, s_{1}$ and $v_{3}, v_{2}, v_{n_{2}}$ cannot contain $K_{3,3}$ as subgraph, it must be $\left(r_{1}, v_{3}\right) \notin E$. Recall that there are no two consecutive vertices of $C$ that are non-adjacent to $r_{1}$, therefore $\left(r_{1}, v_{4}\right) \in E$. Again, since the graph on vertices $r_{1}, t_{1}, s_{1}$ and $v_{4}, v_{2}, v_{n_{2}}$ cannot contain $K_{3,3}$ as subgraph, w.l.o.g it must be $\left(s_{1}, v_{4}\right) \notin E$. But then $t_{1} s_{1} v_{4} C v_{3} r_{1}$ is a hamiltonian path of $G^{c}$ and we are done.

From the above case analysis, it follows that $G^{c}$ has always a Hamiltonian path, as desired.

Clearly, by taking every two consecutive vertices of the Hamiltonian path of $G^{c}$ as a color class, a good 6 -coloring of $G$ is implied. So, we may conclude with the following corollary.

Corollary 1. A planar graph with $n=12$ vertices that does not contain as subgraphs $K_{1,1,9}, K_{1,11}$ and $K_{2,10}$ admits a good 6 -coloring. 


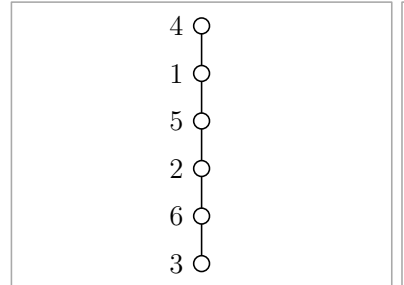

(a) Instance $G=(V, E)$

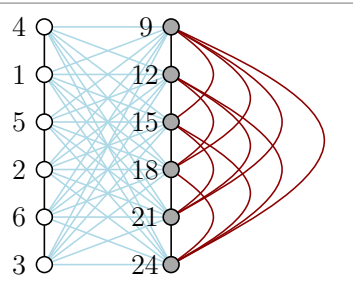

(b) Instance $G^{\prime}=\left(V^{\prime}, E^{\prime}\right)$

Figure 5: (a) An instance of the (3,n)-differential coloring problem for $n=6$; (b) An instance of the $\left(3,2 n^{\prime}\right)$-differential coloring problem constructed based on graph $G$.

\section{NP-completeness Results}

As already stated, all graphs are $(2,2 n)$-differential colorable (recall Lemma 1 ). So, the next reasonable question to ask is whether a given graph is $(3,2 n)$-differential colorable. In the following theorem, we prove that this problem is NP-complete.

Theorem 4. Given a graph $G=(V, E)$ on $n$ vertices, it is $N P$-complete to determine whether $G$ has a $(3,2 n)$-differential coloring.

Proof. The problem is clearly in NP, since a non-deterministic algorithm needs only to guess an assignment of distinct colors (out of $2 n$ available colors) to the vertices of the graph and then it is easy to verify in polynomial time whether this assignment corresponds to a differential coloring of color difference at least 3 .

In order to prove that the problem is NP-hard, we employ a reduction from the $(3, n)$-differential coloring problem, which is known to be NP-complete [6]. More precisely, let $G=(V, E)$ be an instance of the $(3, n)$-differential coloring problem, i.e., graph $G$ is an $n$-vertex graph with vertex set $V=\left\{v_{1}, v_{2}, \ldots, v_{n}\right\}$. We will construct a new graph $G^{\prime}$ with $n^{\prime}=2 n$ vertices, so that $G^{\prime}$ is $\left(3,2 n^{\prime}\right)$-differential colorable if and only if $G$ is $(3, n)$-differential colorable; see Fig. 5

Graph $G^{\prime}=\left(V^{\prime}, E^{\prime}\right)$ is constructed by attaching $n$ new vertices to $G$ that form a clique; see the gray colored vertices of Fig. $5 \mathrm{~b}$ That is, $V^{\prime}=V \cup U$, where $U=$ $\left\{u_{1}, u_{2}, \ldots, u_{n}\right\}$ and $\left(u, u^{\prime}\right) \in E^{\prime}$ for any pair of vertices $u$ and $u^{\prime} \in U$. In addition, for each pair of vertices $v \in V$ and $u \in U$ there is an edge connecting them in $G^{\prime}$, that is $(v, u) \in E^{\prime}$. In other words:

1. the subgraph, say $G_{U}$, of $G^{\prime}$ induced by $U$ is complete, and,

2. the bipartite graph, say $G_{U \times V}$, with bipartition $V$ and $U$ is also complete.

Note that construction of $G^{\prime}$ is known as the join of the two graphs $G$ and $K_{n}$.

First, suppose that $G$ has a $(3, n)$-differential coloring and let $l: V \rightarrow\{1, \ldots, n\}$ be the respective coloring. We compute a coloring $l^{\prime}: V^{\prime} \rightarrow\{1, \ldots, 4 n\}$ of $G^{\prime}$ as follows: (i) $l^{\prime}(v)=l(v)$, for all $v \in V^{\prime} \cap V$ and (ii) $l^{\prime}\left(u_{i}\right)=n+3 i, i=1,2, \ldots, n$. We claim that $l^{\prime}$ is a $\left(3,2 n^{\prime}\right)$-differential coloring of $G^{\prime}$. To realize this first observe that the label difference between any two vertices of $V^{\prime} \cap V$ is at least three, since $G$ 
is $(3, n)$-differential colorable and $l$ is the respective coloring. By the definition of $l^{\prime}$, the label difference between any two vertices of $U$ is exactly three. Finally, the label difference between a vertex in $V^{\prime} \cap V$ and a vertex in $U$ is also at least three, since the vertices of $V^{\prime} \cap V$ are labeled with colors from the set $\{1,2, \ldots, n\}$ and the vertices of $U$ with colors from the set $\{n+3, \ldots, 4 n\}$.

Now, suppose that $G^{\prime}$ is $\left(3,2 n^{\prime}\right)$-differential colorable and let $l^{\prime}: V^{\prime} \rightarrow\left\{1, \ldots, 2 n^{\prime}\right\}$ be the respective coloring (recall that $n^{\prime}=2 n$ ). We next show how to compute the $(3, n)$-differential coloring for $G$. Without loss of generality, let $V=\left\{v_{1}, \ldots v_{n}\right\}$ contain the vertices of $G$, such that $l^{\prime}\left(v_{1}\right)<\ldots<l^{\prime}\left(v_{n}\right)$, and $U=\left\{u_{1}, \ldots u_{n}\right\}$ contain the newly added vertices of $G^{\prime}$, such that $l^{\prime}\left(u_{1}\right)<\ldots<l^{\prime}\left(u_{n}\right)$. Since $G_{U}$ is complete, it follows that the color difference between any two vertices of $U$ is at least three. Similarly, since $G_{U \times V}$ is complete bipartite, the color difference between any two vertices of $U$ and $V$ is also at least three. We claim that $l^{\prime}$ can be converted to an equivalent $\left(3,2 n^{\prime}\right)$-differential coloring for $G^{\prime}$, in which all vertices of $V$ are colored with numbers from 1 to $n$, and all vertices of $U$ with numbers from $n+3$ to $4 n$.

Let $U^{\prime}$ be a maximal set of vertices $\left\{u_{1}, \ldots, u_{j}\right\} \subseteq U$ so that there is no vertex $v \in V$ with $l^{\prime}\left(u_{1}\right)<l^{\prime}(v)<l^{\prime}\left(u_{j}\right)$. If $U^{\prime}=U$ and $l^{\prime}(v)<l^{\prime}\left(u_{1}\right), \forall v \in V$, then our claim trivially holds. If $U^{\prime}=U$ and $l^{\prime}(v)>l^{\prime}\left(u_{j}\right), \forall v \in V$, then we can safely recolor all the vertices in $V^{\prime}$ in reverse order, resulting in a coloring that complies with our claim. Now consider the case where $U^{\prime} \subsetneq U$. Next, we will show how to extend $U^{\prime}$. There is a vertex $v_{k} \in V$ such that $l^{\prime}\left(v_{k}\right)-l^{\prime}\left(u_{j}\right) \geq 3$. Similarly, we define $V^{\prime}=$ $\left\{v_{k}, \ldots, v_{l} \in V\right\}$ to be a maximal set of vertices of $V$, so that $l^{\prime}\left(v_{k}\right)<\ldots<l^{\prime}\left(v_{l}\right)$ and there is no vertex $u \in U$ with $l^{\prime}\left(v_{k}\right)<l^{\prime}(u)<l^{\prime}\left(v_{l}\right)$. Then, we can safely recolor all vertices of $U^{\prime} \cup V^{\prime}$, such that:

1. the relative order of the colors of $U^{\prime}$ and $V^{\prime}$ remains unchanged,

2. the color distance between $v_{l}$ and $u_{1}$ is at least three, and

3. the colors of $U^{\prime}$ are strictly greater than the ones of $V^{\prime}$.

Note that the color difference between $u_{j}$ and $u_{j+1}$ and between $v_{k-1}$ and $v_{k}$ is at least three after recoloring, i.e., $l^{\prime}\left(u_{j+1}\right)-l^{\prime}\left(u_{j}\right) \geq 3$ and $l^{\prime}\left(v_{k}\right)-l^{\prime}\left(v_{k-1}\right) \geq 3$. If we repeat this procedure until $U^{\prime}=U$, then the resulting coloring complies with our claim. Thus, we obtain a $(3, n)$-differential coloring $l$ for $G$ by assigning $l(v)=l^{\prime}(v), \forall v \in V$.

Theorem 5. Given a graph $G=(V, E)$ on $n$ vertices, it is NP-complete to determine whether $G$ has a $(k+1, k n)$-differential coloring, where $k \geq 2$.

Proof. Based on an instance $G=(V, E)$ of the $(k+1, n)$-differential coloring problem, which is known to be NP-complete [6], construct a new graph $G^{\prime}=\left(V^{\prime}, E^{\prime}\right)$ with $n^{\prime}=k n$ vertices, by attaching $n(k-1)$ new vertices to $G$ that form a clique of size $n(k-1)$, as in the proof of Theorem 4 Then, using a similar argument as above, we can show that $G$ has a $(k+1, n)$-differential coloring if and only if $G^{\prime}$ has a $\left(k+1, k n^{\prime}\right)$ differential coloring.

The NP-completeness result of Theorem 4 was for general graphs. In the following, we consider the complexity of the problem for planar graphs. Note that from Lemma2 


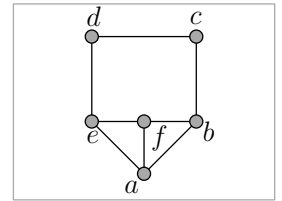

(a) Instance $G$

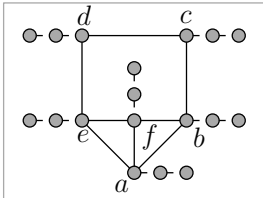

(b) Graph $G^{\prime}$

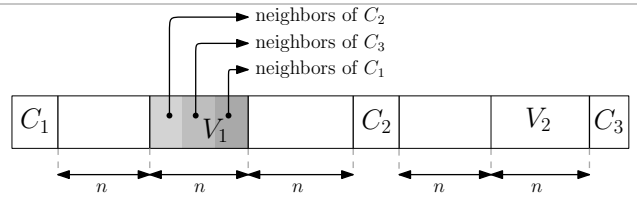

(c) Differential coloring

Figure 6: (a) An instance of the 3-coloring problem; (b) An instance of the $\left(\left\lfloor 2 n^{\prime} / 3\right\rfloor, 2 n^{\prime}\right)$ differential coloring problem constructed based on graph $G$; (c) The $\left(\left\lfloor 2 n^{\prime} / 3\right\rfloor, 2 n^{\prime}\right)$-differential coloring of $G^{\prime}$, in the case where $G$ is 3 -colorable.

and Lemma 3 it follows that the 2-differential chromatic number of a planar graph on $n$ vertices is between $\left\lfloor\frac{n}{3}\right\rfloor+1$ and $\left\lfloor\frac{3 n}{2}\right\rfloor$ (a planar graph is 4 -colorable). The next theorem shows that testing whether a planar graph is $(\lfloor 2 n / 3\rfloor, 2 n)$-differential colorable is NPcomplete. Since this problem can be reduced to the general 2-differential chromatic number problem, it is NP-complete to determine the 2-differential chromatic number even for planar graphs.

Theorem 6. Given an n-vertex planar graph $G=(V, E)$, it is NP-complete to determine if $G$ has a $(\lfloor 2 n / 3\rfloor, 2 n)$-differential coloring.

Proof. The problem is clearly in NP; a non-deterministic algorithm needs only to guess an assignment of distinct colors (out of $2 n$ available colors) to the vertices of the graph and then it is easy to verify in polynomial time whether this assignment corresponds to a differential coloring of minimum color difference at least $\lfloor 2 n / 3\rfloor$.

To prove that the problem is NP-hard, we employ a reduction from the well-known 3 -coloring problem, which is NP-complete for planar graphs [30]. Let $G=(V, E)$ be an instance of the 3-coloring problem, i.e., $G$ is an $n$-vertex planar graph. We will construct a new planar graph $G^{\prime}$ with $n^{\prime}=3 n$ vertices, so that $G^{\prime}$ is $\left(\left\lfloor 2 n^{\prime} / 3\right\rfloor, 2 n^{\prime}\right)$ differential colorable if and only if $G$ is 3 -colorable.

Graph $G^{\prime}=\left(V^{\prime}, E^{\prime}\right)$ is constructed by attaching a path of length two to each vertex of $G$; see Fig. 6a $6 \mathrm{~b}$ Hence, we can assume that $V^{\prime}=V \cup V_{1} \cup V_{2}$, where $V$ is the vertex set of $G, V_{1}$ contains the middle vertex of each 2-vertex path and $V_{2}$ the last vertices. Clearly, $G^{\prime}$ is a planar graph on $n^{\prime}=3 n$ vertices. Since $G$ is a subgraph of $G^{\prime}, G$ is 3-colorable if $G^{\prime}$ is 3-colorable. On the other hand, if $G$ is 3-colorable, then $G^{\prime}$ is also 3-colorable: for each vertex $v \in V$, simply color its neighbors $v_{1} \in V_{1}$ and $v_{2} \in V_{2}$ with two distinct colors different from the color of $v$. Next, we show that $G^{\prime}$ is 3-colorable if and only if $G^{\prime}$ has a $\left(\left\lfloor 2 n^{\prime} / 3\right\rfloor, 2 n^{\prime}\right)$-differential coloring.

First assume that $G^{\prime}$ has a $\left(\left\lfloor 2 n^{\prime} / 3\right\rfloor, 2 n^{\prime}\right)$-differential coloring and let $l: V^{\prime} \rightarrow$ $\left\{1, \ldots, 2 n^{\prime}\right\}$ be the respective coloring. Let $u \in V^{\prime}$ be a vertex of $G^{\prime}$. We assign a color $c(u)$ to $u$ as follows: $c(u)=i$, if $2(i-1) n+1 \leq l(u) \leq 2 i n, i=1,2,3$. Since $l$ is a $\left(\left\lfloor 2 n^{\prime} / 3\right\rfloor, 2 n^{\prime}\right)$-differential coloring, no two vertices with the same color are adjacent. Hence, coloring $c$ is a 3 -coloring for $G^{\prime}$.

Now, consider the case where $G^{\prime}$ is 3-colorable. Let $C_{i} \subseteq V$ be the set of vertices of the input graph $G$ with color $i, i=1,2,3$. Clearly, $C_{1} \cup C_{2} \cup C_{3}=V$. We compute a coloring $l$ of the vertices of graph $G^{\prime}$ as follows (see Fig. 6c): 
- Vertices in $C_{1}$ are assigned colors from 1 to $\left|C_{1}\right|$.

- Vertices in $C_{2}$ are assigned colors from $3 n+\left|C_{1}\right|+1$ to $3 n+\left|C_{1}\right|+\left|C_{2}\right|$.

- Vertices in $C_{3}$ are assigned colors from $5 n+\left|C_{1}\right|+\left|C_{2}\right|+1$ to $5 n+\left|C_{1}\right|+\left|C_{2}\right|+\left|C_{3}\right|$.

- For a vertex $v_{1} \in V_{1}$ that is a neighbor of a vertex $v \in C_{1}, l\left(v_{1}\right)=l(v)+2 n$.

- For a vertex $v_{1} \in V_{1}$ that is a neighbor of a vertex $v \in C_{2}, l\left(v_{1}\right)=l(v)-2 n$.

- For a vertex $v_{1} \in V_{1}$ that is a neighbor of a vertex $v \in C_{3}, l\left(v_{1}\right)=l(v)-4 n$.

- For a vertex $v_{2} \in V_{2}$ that is a neighbor of a vertex $v_{1} \in V_{1}, l\left(v_{2}\right)=l\left(v_{1}\right)+3 n+\left|C_{2}\right|$.

From this construction it follows that the color difference between (i) any two vertices in $G$, (ii) a vertex $v_{1} \in V_{1}$ and its neighbor $v \in V$, and (iii) a vertex $v_{1} \in V_{1}$ and its neighbor $v_{2} \in V_{2}$, is at least $2 n=\left\lfloor\frac{2 n^{\prime}}{3}\right\rfloor$. Thus, $G^{\prime}$ is $\left(\left\lfloor 2 n^{\prime} / 3\right\rfloor, 2 n^{\prime}\right)$-differential colorable.

\section{An ILP for the Maximum k-Differential Coloring Problem}

In this section, we describe an integer linear program (ILP) formulation for the maximum k-differential coloring problem. Recall that an input graph $G$ to the maximum k-differential coloring problem can be easily converted to an input to the maximum 1-differential coloring by creating a disconnected graph $G^{\prime}$ that contains all vertices and edges of $G$ plus $(k-1) n$ isolated vertices. In order to formulate the maximum 1-differential coloring problem as an integer linear program, we introduce for every vertex $v_{i} \in V$ of the input graph $G$ a variable $x_{i}$, which represents the color assigned to vertex $v_{i}$. The 1-differential chromatic number of $G$ is represented by a variable $O P T$, which is maximized in the objective function. The exact formulation is given below. The first two constraints ensure that all vertices are assigned colors from 1 to $n$. The third constraint guarantees that no two vertices are assigned the same color, and the forth constraint maximizes the 1-differential chromatic number of the graph. The first three constraints also guarantee that the variables are assigned integer values.

$$
\begin{array}{llll}
\operatorname{maximize} & O P T & & \\
\text { subject to } & x_{i} & \leq n & \forall v_{i} \in V \\
& x_{i} & \geq 1 & \forall v_{i} \in V \\
& \left|x_{i}-x_{j}\right| \geq 1 & \forall\left(v_{i}, v_{j}\right) \in V^{2} \\
& \left|x_{i}-x_{j}\right| \geq O P T & \forall\left(v_{i}, v_{j}\right) \in E
\end{array}
$$

Note that a constraint that uses the absolute value is of the form $|X| \geq Z$ and therefore can be replaced by two new constraints: (i) $X+M \cdot b \geq Z$ and (ii) $-X+M \cdot(1-b) \geq$ $Z$, where $b$ is a binary variable and $M$ is the maximum value that can be assigned to the sum of the variables, $Z+X$. That is, $M=2 n$. If $b$ is equal to zero, then the two constraints are $X \geq Z$ and $-X+M \geq Z$, with the second constraint always true. On the other hand, if $b$ is equal to one, then the two constraints are $X+M \geq Z$ and $-X \geq Z$, with the first constraint always true. 


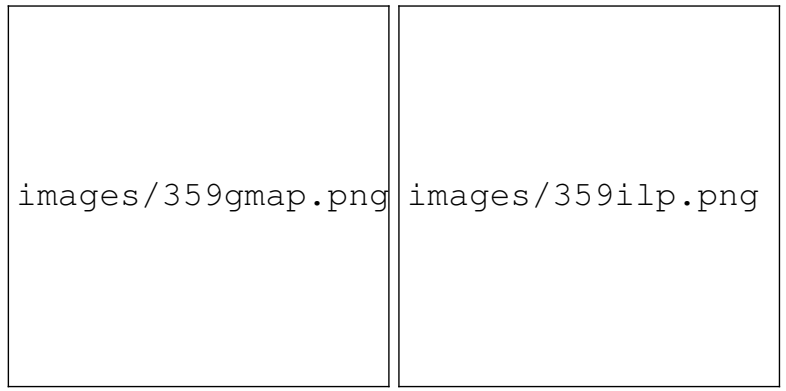

(a) (b)

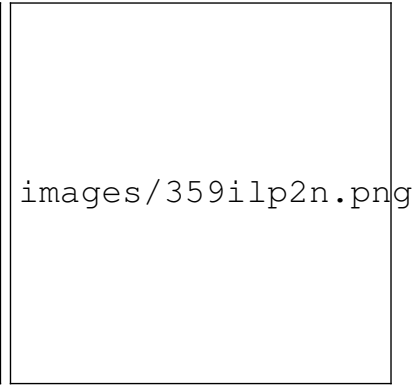

(c)

Figure 7: A map with 16 countries colored by: (a) GMap [21], (b) ILP-n, (c) ILP-2n.

Next, we study two variants of the ILP formulation described above: ILP-n and ILP-2n, which correspond to $k=1$ and $k=2$, and experimentally compare them with the GMap coloring method, which relies on a heuristic based on spectral methods [21]. In order to evaluate the quality of the produced maps, in addition to measuring the minimum color difference between adjacent vertices, we also use the average color difference between adjacent vertices, which for a given coloring $c: V \rightarrow\{1,2, \ldots, k n\}$ of the vertices of a graph $G=(V, E)$ is defined as $\sum_{(i, j) \in E}|c(i)-c(j)| /|E|$. Note that this metric measures the average quality of a coloring, rather than the worst case pairwise value.

\subsection{Experiment's Setup}

We generate a collection of 1,200 synthetic maps and analyze the performance of ILP-n, ILP-2n and GMap, on an Intel Core i5 1.7GHz processor with 8GB RAM, using the CPLEX solver [31]. For each map a country graph $G_{c}=\left(V_{c}, E_{c}\right)$ with $n$ countries is generated using the following procedure, consisting of four steps.

S.1: We generate $10 n$ vertices and place an edge between pairs of vertices $(i, j)$ such that $\left\lfloor\frac{i}{10}\right\rfloor=\left\lfloor\frac{j}{10}\right\rfloor$, with probability 0.5 , thus resulting in a graph $G$ with approximately $n$ clusters.

S.2: More edges are added between all pairs of vertices with probability $p$, where $p$ takes the values $1 / 2,1 / 4, \ldots, 2^{-10}$.

S.3: Ten random graphs are generated for different values of $p$.

S.4: $G$ is used as an input to a map generating algorithm (available as the Graphvi z [32] function gvmap), to obtain a map $M$ with country graph $G_{c}$.

A sample map generated by the aforementioned procedure is shown in Fig.77 Note that the value of $p$ determines the "fragmentation" of the map $M$, i.e., the number of regions in each country, and hence, also affects the number of edges in the country graph. When $p$ is equal to $1 / 2$, the country graph is a nearly complete graph, whereas for $p$ equal to $2^{-10}$, the country graph is nearly a tree. To determine a suitable range for 


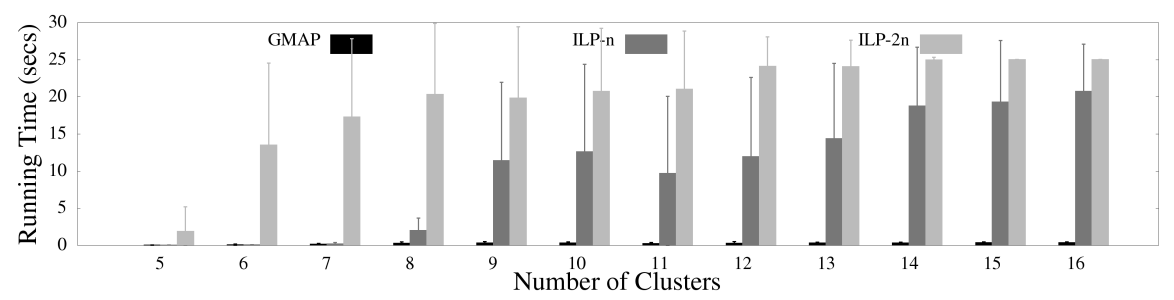

(a) The running time plotted against the number of clusters of the map.

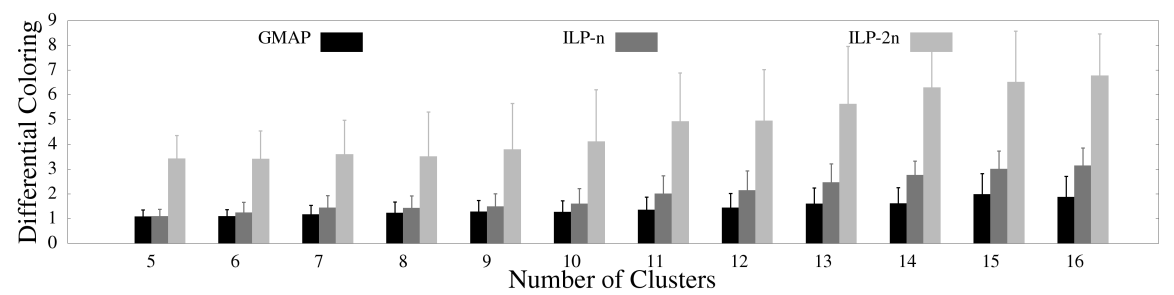

(b) The differential coloring plotted against the number of clusters of the map.

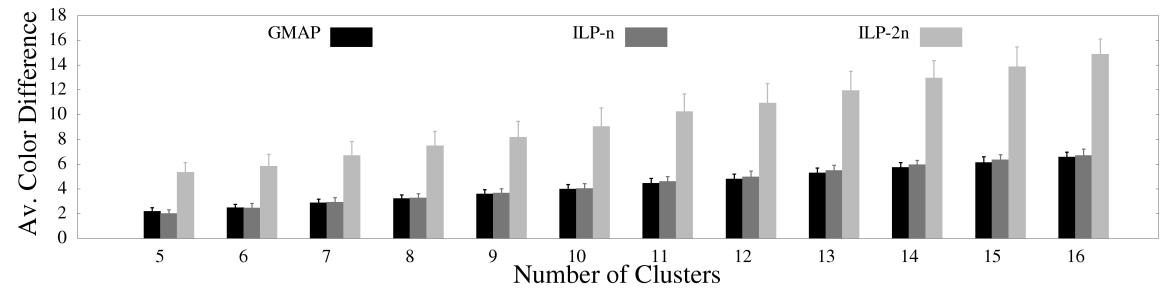

(c) The average color difference plotted against the number of clusters of the map.

Figure 8: Illustration of: (a) running time results, (b) differential coloring performance, and, (c) average color difference.

the number of vertices in the country graph, we evaluated real world datasets, such as those available at gmap. cs . arizona . edu. Even for large graphs with over 1,000 vertices, the country graphs tend to be small, with less than 16 countries.

\subsection{Evaluation Results}

Fig. 8 summarizes the results of our experimental analysis. Since $n$ is ranging from 5 to 16, the running times of both ILP-n and ILP-2n are reasonable, although still much higher than GMap; see Fig. 8a The color assignments produced by ILP-n and GMap are comparable, while the ones produced by ILP-2n are of better minimum and average color difference; see Fig. $8 \mathrm{~b}$ and $8 \mathrm{c}$. It is worth mentioning, though, that in the presence of twice as many colors as the graph's vertices, it is easier to obtain higher color difference between adjacent vertices. However, this high difference comes at the cost of assigning pairs of colors that are more similar to each other for non-adjacent vertices. 


\section{Conclusion and Future Work}

Even though the $(2, n)$-differential coloring is NP-complete for general graphs, in this paper, we gave a complete characterization of bipartite, outerplanar and planar graphs that admit $(2, n)$-differential colorings. Note that these characterizations directly lead to polynomial-time recognition algorithms. We also generalized the differential coloring problem for more colors than the number of vertices in the graph and showed that it is NP-complete to determine whether a general graph admits a $(k+1, k n)$-differential coloring, for $k \geq 2$. Even for planar graphs, the problem of determining whether a graph is $(\lfloor 2 n / 3\rfloor, 2 n)$-differential colorable remains NP-hard. Several related problems are still open:

- Outerplanar and planar graphs form two special cases of the class of so-called degenerate graphs (recall that a $k$-degenerate graph is one in which every subgraph has a vertex of degree at most $k$ ). In particular, outerplanar graphs are 2-degenerate, while the planar ones are 5-degenerate. So, a reasonable question to ask is whether it is possible to characterize which degenerate graphs are $(2, n)$-differential colorable.

- Is it possible to characterize which bipartite, outerplanar or planar graphs are $(3, n)$ differential colorable?

- Extend the characterizations for those planar graphs that admit $(2, n)$-differential colorings to 1-planar graphs, which can be drawn on the plane such that no edge is crossed more than once.

- Extend the results above to $(d, k n)$-differential coloring problems with larger $k>2$.

- As all planar graphs are $\left(\left\lfloor\frac{n}{3}\right\rfloor+1,2 n\right)$-differential colorable, is it possible to characterize which planar graphs are $\left(\left\lfloor\frac{n}{3}\right\rfloor+2,2 n\right)$-differential colorable?

- Since it is NP-complete to determine the 1-differential chromatic number of a planar graph [20], a natural question to ask is whether it is possible to compute in polynomial time the 1-differential chromatic number of an outerplanar graph.

Acknowledgment. The authors would like to thank the anonymous reviewers for their constructive comments.

\section{References}

[1] D. Purves, R. B. Lotto, Why we see what we do: An empirical theory of vision, Sinauer Associates, 2003.

[2] M. Dillencourt, D. Eppstein, M. Goodrich, Choosing colors for geometric graphs via color space embeddings, in: M. Kaufmann, D. Wagner (Eds.), Graph Drawing, Vol. 4372 of LNCS, Springer Berlin Heidelberg, 2007, pp. 294-305.

[3] Y. Hu, S. G. Kobourov, S. Veeramoni, Embedding, clustering and coloring for dynamic maps, J. Graph Algorithms Appl. 18 (1) (2014) 77-109. 
[4] C. Brewer, ColorBrewer - Color Advice for Maps, www. colorbrewer.org.

[5] T. Calamoneri, A. Massini, L. Török, I. Vrt'o, Antibandwidth of complete k-ary trees, Electronic Notes in Discrete Mathematics 24 (2006) 259-266.

[6] J. Y.-T. Leung, O. Vornberger, J. D. Witthoff, On some variants of the bandwidth minimization problem, SIAM Journal on Computing 13 (3) (1984) 650-667.

[7] Z. Miller, D. Pritikin, On the separation number of a graph, Networks 19 (6) (1989) 651-666.

[8] L. Yixun, Y. Jinjiang, The dual bandwidth problem for graphs, Journal of Zhengzhou University (Natural Science) 35 (1) (2003) 1-5.

[9] C. Papadimitriou, The NP-Completeness of the bandwidth minimization problem, Computing 16 (1975) 263-270.

[10] A. Duarte, R. Martí, M. Resende, R. Silva, Grasp with path relinking heuristics for the antibandwidth problem, Networks 58 (3) (2011) 171-189.

[11] R. Bansal, K. Srivastava, Memetic algorithm for the antibandwidth maximization problem, Journal of Heuristics 17 (2011) 39-60.

[12] Y. Hu, S. Kobourov, S. Veeramoni, On maximum differential graph coloring, in: U. Brandes, S. Cornelsen (Eds.), Graph Drawing, Vol. 6502 of LNCS, Springer Berlin Heidelberg, 2011, pp. 274-286.

[13] S. Dobrev, R. Královic, D. Pardubská, L. Török, I. Vrt'o, Antibandwidth and cyclic antibandwidth of hamming graphs, Electronic Notes in Discrete Mathematics 34 (2009) 295-300.

[14] A. Raspaud, H. Schröder, O. Sýkora, L. Török, I. Vrt'o, Antibandwidth and cyclic antibandwidth of meshes and hypercubes, Discrete Math. 309 (11) (2009) 35413552.

[15] X. Wang, X. Wu, S. Dumitrescu, On explicit formulas for bandwidth and antibandwidth of hypercubes, Discrete Applied Mathematics 157 (8) (2009) 1947 1952.

[16] Y. Weili, L. Xiaoxu, Z. Ju, Dual bandwidth of some special trees, Journal of Zhengzhou University (Natural Science) 35 (3) (2003) 16-19.

[17] G. Isaak, Powers of hamiltonian paths in interval graphs, Journal of Graph Theory 27 (1998) 31-38.

[18] W. Hale, Frequency assignment: Theory and applications, Proceedings of the IEEE 68 (12) (1980) 1497 - 1514.

[19] D. Fotakis, S. E. Nikoletseas, V. G. Papadopoulou, P. G. Spirakis, Hardness results and efficient approximations for frequency assignment problems: Radio labelling and radio coloring, Computers and Artificial Intelligence 20 (2). 
[20] M. Bekos, M. Kaufmann, S. Kobourov, S. Veeramoni, A note on maximum differential coloring of planar graphs, Journal of Discrete AlgorithmsAvailable Online.

[21] E. Gansner, Y. Hu, S. Kobourov, Visualizing graphs and clusters as maps, Computer Graphics and Applications, IEEE 30 (6) (2010) 54-66.

[22] R. Diestel, Graph Theory, 4th Edition, Vol. 173 of Graduate texts in mathematics, Springer, 2012.

[23] A. Proskurowski, M. Syso, Efficient vertex- and edge-coloring of outerplanar graphs, SIAM Journal on Algebraic Discrete Methods 7 (1) (1986) 131-136.

[24] M. M. Syso, Characterizations of outerplanar graphs, Discrete Mathematics 26 (1) (1979) 47-53.

[25] K. Appel, W. Haken, Solution of the four color map problem, Scientific American 237 (4) (1977) 108-121.

[26] L. Lovász, J. Pelikán, K. Vesztergombi, Discrete Mathematics: Elementary and Beyond, New York, NY: Springer New York, 2003.

[27] K. Kuratowski, Sur le problme des courbes gauches en topologie, Fund. Math. (in French) 15 (1930) 271-283.

[28] G. Brinkmann, S. Greenberg, C. S. Greenhill, B. D. McKay, R. Thomas, P. Wollan, Generation of simple quadrangulations of the sphere, Discrete Mathematics 305 (1-3) (2005) 33-54.

[29] A. Yang, J. Yuan, On the vertex arboricity of planar graphs of diameter two, Discrete Mathematics 307 (19-20) (2007) 2438-2447.

[30] M. R. Garey, D. S. Johnson, Computers and Intractability: A Guide to the Theory of NP-Completeness, W. H. Freeman \& Co., New York, NY, USA, 1979.

[31] ILOG, Inc, ILOG CPLEX: High-performance software for mathematical programming and optimization, see http://www.ilog.com/products/ cplex/(2006).

[32] J. Ellson, E. Gansner, E. Koutsofios, S. North, G. Woodhull, Graphviz and dynagraph static and dynamic graph drawing tools, in: M. Jünger, P. Mutzel (Eds.), Graph Drawing Software, Mathematics and Visualization, Springer Berlin Heidelberg, 2004, pp. 127-148. 